\title{
ON ISOMETRY GROUPS AND MAXIMAL SYMMETRY
}

\section{VALENTIN FERENCZI and CHRISTIAN ROSENDAL}

In memory of Greg Hjorth (1963-2011) and Nigel Kalton (1946-2010)

\begin{abstract}
We study problems of maximal symmetry in Banach spaces. This is done by providing an analysis of the structure of small subgroups of the general linear group $\mathrm{GL}(X)$, where $X$ is a separable reflexive Banach space. In particular, we provide the first known example of a Banach space $X$ without any equivalent maximal norm, or equivalently such that $\mathrm{GL}(X)$ contains no maximal bounded subgroup. Moreover, this space $X$ may be chosen to be super-reflexive.
\end{abstract}

\section{Contents}

1. Introduction . . . . . . . . . . . . . . . . . . . 1771

2. Notation and complexifications . . . . . . . . . . . . . . . . . . . 1777

3. Bounded subgroups of $\mathrm{GL}(X)$. . . . . . . . . . . . . . . . . . . . . 1779

4. Decompositions of separable reflexive spaces by isometries . . . . . . . 1791

5. Groups of finite-dimensional isometries . . . . . . . . . . . . . . . . 1804

6. Spaces with a small algebra of operators . . . . . . . . . . . . . . . . 1811

7. Maximality and transitivity in spaces with few operators . . . . . . . . 1817

8. Questions and further comments . . . . . . . . . . . . . . . . . 1821

References . . . . . . . . . . . . . . . . . . . . 1827

\section{Introduction}

\subsection{Maximal norms and the problems of Mazur and Dixmier}

Two outstanding problems of functional analysis are Mazur's rotation problem, ask-

DUKE MATHEMATICAL JOURNAL

\footnotetext{
Vol. 162, No. 10, () 2013 DOI 10.1215/00127094-2322898

Received 19 March 2012. Revision received 15 October 2012.

2010 Mathematics Subject Classification. Primary 22F50; Secondary 03E15, 46B03, 46B04.

Ferenczi's work partially supported by Conselho Nacional de Desenvolvimento Cientifico e Tecnológico (Brazil) project 452068/2010-0, and by Fundação de Amparo a Pesquisa do Estado de São Paulo (Brazil) projects 2010/05182-1 and 2010/17493-1.

Rosendal's work supported by National Science Foundation grant DMS-0901405.
} 
ing whether any separable Banach space whose isometry group ${ }^{\dagger}$ acts transitively on the sphere must be a Hilbert space, and Dixmier's unitarizability problem, asking whether any countable group, all of whose bounded representations on Hilbert space are unitarizable, must be amenable. Although these problems do not on the surface seem to be related, they both point to common geometric aspects of Hilbert space that are far from being well understood.

Mazur's problem, which can be found in Banach's classical work (see [7]), is perhaps best understood as two separate problems, both of which remain open to this day.

\section{Problem 1.1 (Mazur's rotation problem, first part)}

Suppose that $X$ is a separable Banach space whose isometry group acts transitively on the sphere $S_{X}$. Is $X$ Hilbertian, that is, isomorphic to the separable Hilbert space $\mathscr{H}$ ?

We remark that, in order for the problem to be nontrivial, the separability condition in the hypothesis is necessary. This is because the isometry group of $L_{p}$ induces a dense orbit on the sphere (see [36], [58]) and thus the isometry group will act transitively on any ultrapower of $L_{p}$, which itself is an $L_{p}$-space.

\section{Problem 1.2 (Mazur's rotation problem, second part)}

Suppose that $\||\cdot| \mid$ is an equivalent norm on $\mathscr{H} \operatorname{such}$ that $\operatorname{Isom}(\mathscr{H},\||\||)$ acts transitively on the unit sphere $S_{\mathscr{H}}^{\|\cdot \cdot\| \|}$. Is $\||\cdot|||$ necessarily Euclidean?

Establishing a vocabulary to study these problems, Pełczyński and Rolewicz [52] (see also Rolewicz [58]) defined the norm $\|\cdot\|$ of a Banach space $X$ to be maximal if whenever $\|\cdot \mid\|$ is an equivalent norm on $X$ with

$$
\operatorname{Isom}(X,\|\cdot\|) \leq \operatorname{Isom}(X,\|\mid \cdot\|)
$$

that is, $\operatorname{Isom}(X,\|\cdot\|)$ being a subgroup of $\operatorname{Isom}(X,\|\cdot \mid\|)$, then

$$
\operatorname{Isom}(X,\|\cdot\|)=\operatorname{Isom}(X,\|\cdot\| \|) \text {. }
$$

Also, the norm is transitive if the isometry group acts transitively on the unit sphere.

Thus, a norm is maximal if one cannot replace it by another equivalent norm that has strictly more isometries, or, more suggestively, if the unit ball $B_{X}^{\|\cdot\|}$ is a maximally symmetric body in $X$. Note also that, if $\|\cdot\|$ is transitive, then the unit sphere $S_{X}^{\|\cdot\|}$ is the orbit of a single point $x \in S_{X}^{\|\cdot\|}$ under the action of $\operatorname{Isom}(X,\|\cdot\|)$, and so any

\footnotetext{
${ }^{\dagger}$ Since we are treating only groups of linear isometries, henceforth, any isometry will be assumed to be invertible and linear. Moreover, we let $\operatorname{Isom}(X,\|\cdot\|)$ denote the group of all invertible linear isometries of a given Banach space $(X,\|\cdot\|)$, while $\operatorname{GL}(X)$ denotes the group of all bounded invertible operators on $X$.
} 
proper supergroup $G$ of $\operatorname{Isom}(X,\|\cdot\|)$ inside of $\operatorname{GL}(X)$ must send $x$ to some point $\lambda x$ for $|\lambda| \neq 1$, from which it follows that $G$ cannot be a group of isometries for any norm. So transitivity implies maximality, and thus the standard Euclidean norm $\|\cdot\|_{2}$ is maximal on $\mathscr{H}$. Also, Mazur [47] himself showed that any transitive norm on a finite-dimensional space is, in effect, Euclidean (see the survey papers by CabelloSánchez [13] and Becerra Guerrero and Rodríguez-Palacios [8] for more information on the rotation problem and maximal norms, and also [12] for results in the purely metric case).

Another way of understanding these concepts, pointing toward the unitarizability problem of Dixmier, is by considering the $G$-invariant norms corresponding to a bounded subgroup $G \leq \operatorname{GL}(X)$. Here $G$ is bounded if $\|G\|=\sup _{T \in G}\|T\|<\infty$. Note first that if $G$ is bounded, then

$$
\|x\|=\sup _{T \in G}\|T x\|
$$

defines an equivalent $G$-invariant norm on $X$; that is, $G \leq \operatorname{Isom}(X,\|\cdot\| \|)$. Moreover, if $\|\cdot\|$ is uniformly convex, then so is $\|\cdot\| \|$ (see, e.g., [6, Proposition 2.3]). However, if $X=\mathscr{H}$ and $\|\cdot\|$ is Euclidean, that is, induced by an inner product, then $\|\cdot \mid\|$ will not, in general, be Euclidean. The question of which bounded subgroups of GL( $\mathscr{H})$ admit invariant Euclidean norms has a long history. It is a classical result of representation theory dating back to the beginning of the twentieth century that if $G \leq \operatorname{GL}\left(\mathbb{C}^{n}\right)$ is a bounded subgroup, then there is a $G$-invariant inner product, thus inducing a $G$-invariant Euclidean norm. Also, in the 1930s, Szókefalvi-Nagy [65, Theorem I] showed that any bounded representation $\pi: \mathbb{Z} \rightarrow \mathrm{GL}(\mathscr{H})$ is unitarizable, that is, $\mathscr{H}$ admits an equivalent $\pi(\mathbb{Z})$-invariant inner product and, with the advent of amenability in the 1940s, this was extended by Day [18] and Dixmier [22] to any bounded representation of an amenable topological group, via averaging over an invariant mean. In the opposite direction, Ehrenpreis and Mautner [24] constructed a nonunitarizable bounded representation of $\mathrm{SL}_{2}(\mathbb{R})$ on $\mathscr{H}$, and the group $\mathrm{SL}_{2}(\mathbb{R})$ was later replaced by any countable group containing the free group $\mathbb{F}_{2}$. However, since, by a result of Ol'šhanskiı̌ [51], $\mathbb{F}_{2}$ does not embed into all nonamenable countable groups, the question of whether the result of Szókefalvi-Nagy, Day, and Dixmier reverses still remains pertinent.

\section{Problem 1.3 (Dixmier's unitarizability problem)}

Suppose that $\Gamma$ is a countable group all of whose bounded representations on $\mathscr{H}$ are unitarizable. Is $\Gamma$ amenable?

Nevertheless, though not every bounded representation of $\mathbb{F}_{2}$ in $\operatorname{GL}(\mathcal{H})$ is unitarizable, it still seems to be unknown whether all of its bounded representations 
admit equivalent invariant maximal or even transitive norms. And similarly, while the second part of the rotation problem asks whether any equivalent transitive norm on $\mathscr{H}$ is Euclidean, the stronger question of whether any equivalent maximal norm is Euclidean also remains open. Of course, the counterexample of Ehrenpreis and Mautner limits how much of these two questions can hold simultaneously (see the recent survey by Pisier [54] and also [25] and [49] for the current status of Dixmier's problem).

In a more general direction, the work of Pełczyński and Rolewicz led people to investigate which spaces have maximal norms. Since any bounded subgroup $G \leq$ $\mathrm{GL}(X)$ is contained in a group of isometries for an equivalent norm, one observes that a norm $\|\cdot\|$ is maximal if and only if the corresponding isometry group is a maximal bounded subgroup of $\mathrm{GL}(X)$. Thus, in analogy with the existence of maximal compact subgroups of semisimple Lie groups, it is natural to suspect that a judicious choice of smoothing procedures on a space $X$ could eventually lead to a most symmetric norm, which then would be maximal on $X$. But, even so, fundamental questions on maximal norms have remained open, including notably the long-standing problem, formulated by Wood in [68], of whether any Banach space $X$ admits an equivalent maximal norm. In fact, even the question of whether any bounded $G \leq \mathrm{GL}(X)$ is contained in a maximal bounded subgroup was hitherto left unresolved. Our main result answers these, as well as another problem of Deville, Godefroy, and Zizler [21], in the negative.

\section{THEOREM 1.4}

There is a separable super-reflexive Banach space $X$ such that $\mathrm{GL}(X)$ contains no maximal bounded subgroups, that is, $X$ has no equivalent maximal norm.

\subsection{Nontrivial isometries of Banach spaces}

A second motivation and a source of tools for our work comes from the seminal construction of Gowers and Maurey [35] of a space GM with a small algebra of operators, namely, such that any operator on GM is a strictly singular perturbation of a scalar multiple of the identity map. The strongest result to date in this direction, due to Argyros and Haydon [4], is the construction of a Banach space AH on which every operator is a compact perturbation of a scalar multiple of the identity. Furthermore, since $\mathrm{AH}$ has a Schauder basis, every compact operator is a limit of operators of finite rank.

These results largely answer the question of whether every Banach space admits nontrivial operators, but one can ask the same question for isometries, that is, does every Banach space admit a nontrivial surjective isometry? After partial answers by Semenev and Skorik [60] and Bellenot [9], one version of this question was answered 
in the negative by Jarosz [39]. Jarosz proved that any real or complex Banach space admits an equivalent norm with only trivial isometries, namely, such that any surjective isometry is a scalar multiple of the identity, $\lambda \mathrm{Id}$, for $|\lambda|=1$. Thus, no isomorphic property of a space can force the existence of a nontrivial surjective linear isometry.

Of course, this does not prevent the group of isometries to be extremely nontrivial in some other equivalent norm. So one would like results relating the size of the isometry group $\operatorname{Isom}(X,\|\cdot\|)$, for any equivalent norm $\|\cdot\|$, with the isomorphic structure of $X$. Let us first remark that any infinite-dimensional Banach space $X$ can always be equivalently renormed such that $X=F \oplus_{1} H$, where $F$ is a finite-dimensional Euclidean space. So, in this case, $\operatorname{Isom}(X)$ will at least contain a subgroup isomorphic to $\operatorname{Isom}(F)$. Actually, if $X$ is a separable, infinite-dimensional, real space and $G$ is a finite group, then it is possible to find an equivalent norm for which $\{-1,1\} \times G$ is isomorphic to the group of isometries on $X$ (see [29], [63]).

Thus, allowing for renormings, we need a less restrictive concept of when an isometry is trivial.

\section{Definition 1.5}

A bounded subgroup $G \leq \mathrm{GL}(X)$ acts nearly trivially on $X$ if there is a $G$-invariant decomposition $X=F \oplus H$, where $F$ is finite-dimensional and where $G$ acts by trivial isometries on $H$.

As an initial step toward Theorem 1.4, we show that in a certain class of spaces, each individual isometry acts nearly trivially. For that, we need to improve on some earlier work of Räbiger and Ricker [55], [56]. By their results, any isometry of a socalled hereditarily indecomposable complex Banach space is a compact perturbation of a scalar multiple of the identity, but this can be improved as follows.

\section{THEOREM 1.6}

Let $X$ be a Banach space containing no unconditional basic sequence and on which every operator is of the form $\lambda \mathrm{Id}+S$, for $S$ strictly singular. Then each individual isometry acts nearly trivially on $X$.

The main problem is then to investigate when we can proceed from single isometries acting nearly trivially to an understanding of the global structure of the isometry group Isom $(X)$. Disregarding for the moment the scalar multiples of the identity on $X$, we consider the automorphisms of $X$ that individually act nearly trivially on $X$. For this, we let $\mathrm{GL}_{f}(X)$ denote the subgroup of $\mathrm{GL}(X)$ consisting of all automorphisms of the form

$$
\mathrm{Id}+A
$$


where $A$ is a finite-rank operator on $X$. We then establish, in the case of separable reflexive $X$, the structure of bounded subgroups of $\mathrm{GL}_{f}(X)$ that are strongly closed in $\mathrm{GL}(X)$. The following statement refers to the strong operator topology on $G$.

\section{THEOREM 1.7}

Suppose that $X$ is a separable reflexive Banach space and that $G \leq \mathrm{GL}_{f}(X)$ is bounded and strongly closed in $\mathrm{GL}(X)$. Let also $G_{0} \leq G$ denote the connected component of the identity in $G$.

Then $G_{0}$ acts nearly trivially on $X$ and therefore is a compact Lie group. Moreover, $G_{0}$ is open in $G$, while $G / G_{0}$ is a countable, locally finite and thus amenable group. It follows that $G$ is an amenable Lie group.

Furthermore, $X$ admits a $G$-invariant decomposition $X=X_{1} \oplus X_{2} \oplus X_{3} \oplus X_{4}$, where

(1) no nonzero point of $X_{1}$ has a relatively compact $G$-orbit;

(2) every $G$-orbit on $X_{2} \oplus X_{3} \oplus X_{4}$ is relatively compact;

(3) $X_{4}$ is the subspace of points which are fixed by $G$;

(4) $X_{3}$ is finite-dimensional or has a finite-dimensional decomposition;

(5) $\quad X_{2}$ is finite-dimensional and $X_{1} \oplus X_{3} \oplus X_{4}$ is the subspace of points which are fixed by $G_{0}$;

(6) if $X_{1}=\{0\}$, then $G$ acts nearly trivially on $X$;

(7) if $X_{1} \neq\{0\}$, then $X_{1}$ is infinite-dimensional, has a finite-dimensional decomposition, and admits a $G$-invariant Schauder decomposition (possibly with finitely many terms),

$$
X_{1}=Y_{1} \oplus Y_{2} \oplus \cdots,
$$

where every $Y_{i}$ has a Schauder basis.

Combining Theorem 1.6 with a somewhat simpler version of Theorem 1.7 along with an earlier construction of a super-reflexive hereditarily indecomposable space due to the first author [27], we are able to conclude the following result from which Theorem 1.4 is easily obtained.

\section{THEOREM 1.8}

Let $X$ be a separable, reflexive, hereditarily indecomposable, complex Banach space without a Schauder basis. Then, for any equivalent norm on $X$, the group of isometries acts nearly trivially on $X$.

Moreover, there are super-reflexive spaces satisfying these hypotheses.

Note that if $\operatorname{Isom}(X,\|\cdot\|)$ acts nearly trivially on $X$, it is isomorphic to a closed subgroup of a finite-dimensional unitary group and thus is itself a compact Lie group. 
Now, as shown by von Neumann and Wigner [67], there are countable minimally almost periodic groups, that is, countable discrete groups admitting no nontrivial finite-dimensional unitary representations or, equivalently, such that any homomorphism into a compact Hausdorff group is trivial. An example of such a group is $\operatorname{PSL}_{2}(\mathbb{Q})$, that is, the quotient of the group $\mathrm{SL}_{2}(\mathbb{Q})$ of rational $(2 \times 2)$-matrices with determinant 1 by its center $\{I,-I\}$. By Theorem 1.8 , it follows immediately that no minimally almost periodic group admits a bounded linear representation on a separable, reflexive, hereditarily indecomposable, complex Banach space without a Schauder basis.

\section{Notation and complexifications}

\subsection{Some notation and terminology}

For a Banach space $X$, we denote by $\mathscr{L}(X)$ the algebra of continuous linear operators on $X$, by $\mathrm{GL}(X)$ the general linear group of $X$, that is, the group of all continuous linear automorphisms of $X$, and by $\operatorname{Isom}(X)$ the group of surjective linear isometries of $X$. In the remainder of the paper, unless explicitly stated otherwise, an isometry of $X$ is always assumed to be surjective, so we will not state this hypothesis explicitly. We also denote the unit sphere of $X$ by $S_{X}$ and the closed unit ball by $B_{X}$. When $x_{1}, \ldots, x_{n}$ is a sequence of vectors in $X$, we let $\left[x_{1}, \ldots, x_{n}\right]$ denote the linear subspace spanned by the $x_{i}$.

If $X$ is a complex space and $T$ is an operator on $X, \sigma(T)$ denotes the spectrum of $T$ and one observes that $\sigma(T)$ is a subset of the unit circle $\mathbb{T}$ whenever $T$ is an isometry. On the other hand, if $T$ is compact, then $T$ is a Riesz operator, which means that $\sigma(T)$ is either a finite sequence of eigenvalues with finite multiplicity together with 0 , or an infinite converging sequence of such eigenvalues together with the limit point 0 .

As our proofs use methods from representation theory, spectral theory, and renorming theory, as well as general Banach space theory, we have tried to give selfcontained and detailed proofs of our results in order for the paper to remain readable for a larger audience. Of course, some of the background material is true in a broader setting, which may be easily found by consulting the literature. Our main references will be Dunford and Schwartz [23] for spectral theory, Deville, Godefroy, and Zizler [21] for renorming theory, and Lindenstrauss and Tzafriri [44], [45] and Benyamini and Lindenstrauss [10] for general Banach space and some operator theory. We also recommend Fleming and Jamison [31], especially Chapter 12, for more information on isometries of Banach spaces. 


\subsection{Complex spaces versus real spaces}

Our main results will be valid both in the real and the complex settings, though different techniques will sometimes be needed to cover each separate case.

For the part of our demonstrations using spectral theory, as is classical, we first prove our results in the complex case and thereafter use complexification to extend them to the real case. We recall briefly how this is done and the links that exist between a real space and its complexification.

If $X$ is a real Banach space with norm $\|\cdot\|$, the complexification $\hat{X}$ of $X$ is defined as the Cartesian square $X \times X$, whose elements are written $x+i y$ rather than $(x, y)$ for $x, y \in X$, equipped with the complex scalar multiplication given by

$$
(a+i b) \cdot(x+i y)=a x-b y+i(b x+a y),
$$

for $a, b \in \mathbb{R}$ and $x, y \in X$, and with the equivalent norm

$$
\|x+i y\|=\sup _{\theta \in[0,2 \pi]}\left\|e^{i \theta}(x+i y)\right\|_{2},
$$

where

$$
\|x+i y\|_{2}=\sqrt{\|x\|^{2}+\|y\|^{2}} .
$$

Any operator in $\mathscr{L}(\hat{X})$ may be written of the form $T+i U$, where $T, U$ belong to $\mathscr{L}(X)$, that is, for $x, y \in X$,

$$
(T+i U)(x+i y)=T x-U y+i(U x+T y) .
$$

We denote by $c$ the natural isometric homomorphism from $\mathscr{L}(X)$ into $\mathscr{L}(\hat{X})$ associating to $T$ the operator $\hat{T}$ defined by

$$
\hat{T}=T+i 0 .
$$

It is then straightforward to check that the image by $c$ of an automorphism (resp., isometry, finite-rank perturbation of the identity, compact perturbation of the identity) of $X$ is an automorphism (resp., isometry, finite-rank perturbation of the identity, compact perturbation of the identity) of $\hat{X}$. In other words, the map $c$ provides an embedding of natural subgroups of $\operatorname{GL}(X)$ into their counterparts in $\operatorname{GL}(\hat{X})$.

Conversely, in renorming theory it is usually assumed that the spaces are real. One obtains renorming results on a complex space $X$ simply by considering only its $\mathbb{R}$-linear structure which defines a real space $X_{\mathbb{R}}$. It is well known that many isomorphic properties of a space do not depend on it being seen as real or complex, and we recall here briefly the facts that we will rely on later.

First, by [10], a space $X$ has the Radon-Nikodym property (RNP) if and only if every Lipschitz function from $\mathbb{R}$ into $X$ is differentiable almost everywhere, which 
only depends on the $\mathbb{R}$-linear structure of $X$. A space $X$ is reflexive if and only if the closed unit ball of $X$ is weakly sequentially compact, and hence $X$ is reflexive if and only if $X_{\mathbb{R}}$ is reflexive.

Moreover, the map $\phi \mapsto \operatorname{Re}(\phi)$ is an $\mathbb{R}$-linear isometry from $X^{*}$ onto $\left(X_{\mathbb{R}}\right)^{*}$ with inverse $\psi \mapsto \phi$ defined by

$$
\phi(x)=\psi(x)-i \psi(i x)
$$

So the dual norms on $X^{*}$ and $\left(X_{\mathbb{R}}\right)^{*}$ coincide up to this identification and $X$ has separable dual if and only if $X_{\mathbb{R}}$ has. Likewise, if $\phi$ is a $\mathbb{C}$-support functional for $x_{0} \in S_{X}$ (i.e., $\phi\left(x_{0}\right)=\|\phi\|=1$ ), then $\operatorname{Re}(\phi)$ is an $\mathbb{R}$-support functional for $x_{0}$. Thus, the above identification shows that if in a complex space a point $x$ in $S_{X}$ has a unique $\mathbb{R}$-support functional, then it has a unique $\mathbb{C}$-support functional, which, in particular, happens when the norm on $X$ is Gâteaux differentiable (see [21]).

\section{Bounded subgroups of $\mathrm{GL}(X)$}

In this section, we will review some general facts about bounded subgroups of $\operatorname{GL}(X)$. While all of the material here, apart from Theorems 3.4 and 3.11, is well known, it may not be available in any single source.

\subsection{Topologies on $\mathrm{GL}(X)$}

Suppose that $X$ is a real or complex Banach space and that $G \leq \operatorname{GL}(X)$ is a weakly bounded subgroup, that is, such that for any $x \in X$ and $\phi \in X^{*}$,

$$
\sup _{T \in G}|\phi(T x)|<\infty
$$

Then, by the uniform boundedness principle, $G$ is actually norm bounded, that is,

$$
\|G\|=\sup _{T \in G}\|T\|<\infty .
$$

So without ambiguity we can simply refer to $G$ as a bounded subgroup of GL( $X)$.

Note that if $G$ is bounded, then

$$
\|x\|=\sup _{T \in G}\|T x\|
$$

is an equivalent norm on $X$ such that $G$ acts by isometries on $(X,\|\cdot\|)$. Therefore, bounded subgroups of $\mathrm{GL}(X)$ are simply subgroups of the isometry groups of equivalent norms on $X$.

Let us also stress the fact that, although the operator norm changes when $X$ is given an equivalent norm, the norm, weak, and strong operator topologies on GL $(X)$ remain unaltered. 
Recall that if $X$ is separable, the isometry group, $\operatorname{Isom}(X)$, is a Polish group in the strong operator topology, that is, a separable topological group whose topology can be induced by a complete metric. Since any strongly closed bounded subgroup $G \leq \mathrm{GL}(X)$ can be seen as a strongly closed subgroup of $\operatorname{Isom}(X)$ for an equivalent norm on $X$, provided that $X$ is separable, we find that $G$ is a closed subgroup of a Polish group and hence is Polish itself.

Note also that the norm induces an invariant, complete metric on $\operatorname{Isom}(X)$, that is, $\|T S U-T R U\|=\|S-R\|$ for all $T, S, R, U \in \operatorname{Isom}(X)$, and so $\operatorname{Isom}(X)$ and, similarly, any bounded subgroup $G \leq \mathrm{GL}(X)$, is a SIN group in the norm topology, that is, admits a neighborhood basis at the identity consisting of conjugacy-invariant sets.

Of course, even when $X$ is separable, the norm topology is usually nonseparable on $\operatorname{Isom}(X)$, but, as we will see, for certain small subgroups of $\operatorname{GL}(X)$ it coincides with the strong operator topology, which allows for an interesting combination of different techniques.

Note that if $X$ is separable, we can choose a dense subset of the unit sphere $\left\{x_{n}\right\} \subseteq S_{X}$ and corresponding support functionals $\left\{\phi_{n}\right\} \subseteq S_{X^{*}}, \phi_{n}\left(x_{n}\right)=1$. Using these, we can write the closed unit ball $B_{X}$ as

$$
B_{X}=\bigcap_{n, m}\left\{x \in X \mid \phi_{n}(x)<1+1 / m\right\}
$$

which shows that $B_{X}$ is a countable intersection of open half-spaces in $X$ and similarly for any other closed ball in $X$. Thus, if $\pi: G \rightarrow \operatorname{GL}(X)$ is a bounded, weakly continuous representation of a Polish group $G$, then for any $x \in X$ and $\epsilon>0$, the set

$$
\{g \in G \mid \pi(g) x \in \overline{B(x, \epsilon)}\}
$$

is a countable intersection of open sets in $G$ and hence is Borel. It follows that $\pi$ is a Borel homomorphism from a Polish group into the separable group $(\pi(G)$, SOT) and hence, by Pettis's theorem (see [41, (9.10)]), $\pi$ is strongly continuous.

\section{PROPOSITION 3.1}

Let $X$ be a separable Banach space, and let $\pi: G \rightarrow \mathrm{GL}(X)$ be a bounded, weakly continuous representation of a Polish group $G$. Then $\pi$ is strongly continuous.

In fact, if $X^{*}$ is separable, the weak and strong operator topologies coincide on any bounded subgroup $G \leq \mathrm{GL}(X)$ (see, e.g., [48] for more information on this).

What is more important is that if $\pi: G \rightarrow \operatorname{GL}(X)$ is a strongly continuous bounded representation of a Polish group, the induced dual representation $\pi^{*}: G \rightarrow$ $\mathrm{GL}\left(X^{*}\right)$ is in general only ultraweakly continuous, that is, $\pi^{*}\left(g_{i}\right)(\phi) \underset{\text { weak }^{*}}{\longrightarrow} \pi^{*}(g)(\phi)$ 
for $\phi \in X^{*}$ and $g_{i} \rightarrow g$. Of course, if $X$ is separable and reflexive, this means that $\pi^{*}$ is weakly continuous and thus also strongly continuous.

In the following, the default topology on $\operatorname{GL}(X)$ and its subgroups is the strong operator topology. So, unless otherwise stated, all statements refer to this topology. Moreover, if $G \leq \mathrm{GL}(X)$, then $\bar{G}^{\text {SOT }}$ refers to the strong closure in $\operatorname{GL}(X)$ and not in $\mathscr{L}(X)$. This is important, since even a bounded subgroup that is strongly closed in $\operatorname{GL}(X)$ may not be strongly closed in $\mathscr{L}(X)$; for example, the unitary group of infinite-dimensional Hilbert space, $U\left(\ell_{2}\right)$, is not strongly closed in $\mathscr{L}\left(\ell_{2}\right)$. This is in opposition to the well-known fact that any bounded subgroup that is norm closed in $\operatorname{GL}(X)$ is also norm closed in $\mathscr{L}(X)$. However, for potentially unbounded $G \leq$ $\mathrm{GL}(X)$, we let $\bar{G}^{\|\cdot\|}$ denote the norm closure of $G$ in $\operatorname{GL}(X)$.

A topological group $G$ is said to be precompact if any nonempty open set $\mathcal{U} \subseteq$ $G$ covers $G$ by finitely many left translates; that is, $G=A U$ for some finite set $A \subseteq G$. For Polish groups, this is equivalent to being compact, but, for example, any nonclosed subgroup of a compact Polish group is only precompact and not compact.

For the next proposition, we recall that a vector $x \in X$ is said to be almost periodic with respect to some $G \leq \operatorname{GL}(X)$ if the $G$-orbit of $x$ is relatively compact or, equivalently, totally bounded in $X$ (i.e., is covered by finitely many $\epsilon$-balls for all $\epsilon>0$ ). In analogy with this, $G$ is said to be almost periodic if every $x \in X$ is almost periodic.

\section{PROPOSITION 3.2}

Suppose that $X$ is a Banach space and that $G \leq \mathrm{GL}(X)$ is a bounded subgroup. Then the following are equivalent:

(1) $\quad X$ is the closed linear span of its finite-dimensional irreducible subspaces;

(2) $G$ is almost periodic;

(3) $G$ is precompact;

(4) $\bar{G}^{\text {SOT }}$ is compact.

\section{Proof}

$(1) \Rightarrow(2)$ : Using that relative compactness is equivalent to total boundedness, it is an easy exercise to see that the set of almost periodic points form a closed linear subspace and, moreover, since $G$ is bounded, any finite-dimensional $G$-invariant subspace is contained in the set of almost periodic points. So (2) follows from (1).

$(2) \Rightarrow(3)$ : Note that if $G$ is not precompact in the strong operator topology, then there is an open neighborhood $\mathcal{U}$ of Id that does not cover $G$ by a finite number of left translates. It follows that we can find a finite sequence of normalized vectors $x_{1}, \ldots, x_{n} \in X, \epsilon>0$ and an infinite set $\mathcal{A} \subseteq G$ such that for distinct $T, U \in \mathcal{A}$ there is $i$ with $\left\|T x_{i}-U x_{i}\right\|>\epsilon$. But then, by the infinite version of Ramsey's theorem (see 
[57]), we may find some $i$ and some infinite subset $\mathscr{B}$ of $\mathcal{A}$ such that $\left\|T x_{i}-U x_{i}\right\|>\epsilon$ whenever $T, U \in \mathscr{B}$ are distinct, which shows that the $G$-orbit of $x_{i}$ is not relatively compact.

(3) $\Rightarrow(4)$ : If $G$ is precompact, $\bar{G}^{\text {SOT }}$ is easily seen to be precompact. It follows that every $\bar{G}^{\text {SOT }}$-orbit is totally bounded, that is, relatively compact. So, to see that $\bar{G}^{\text {SOT }}$ is compact, let $\left(g_{i}\right)$ be a net in $\bar{G}^{\text {SOT }}$, and pick a subnet $\left(h_{j}\right)$ such that, for every $x \in X,\left(h_{j} x\right)$ and $\left(h_{j}^{-1} x\right)$ converge to some $T x$ and $S x$, respectively. It follows that $S=T^{-1} \in \bar{G}^{\text {SOT }}$, and so $\left(h_{j}\right)$ converges in the strong operator topology to $T \in \bar{G}^{\text {SOT }}$. Since every net has a convergent subnet, $\bar{G}^{\text {SOT }}$ is compact.

(4) $\Rightarrow(1)$ : Suppose that $\bar{G}^{\text {SOT }} \leq \mathrm{GL}(X)$ is compact, and consider the tautological strongly continuous representation $\pi: \bar{G}^{\text {SOT }} \rightarrow \mathrm{GL}(X)$. By a result going back to at least Shiga [61, Theorem 2], since $\bar{G}^{\text {SOT }}$ is compact, $X$ is the closed linear span of its finite-dimensional irreducible subspaces, that is, minimal nontrivial $G$-invariant subspaces, and so (1) follows. (Note that the result of Shiga is stated only for the complex case in [61], but the real case follows from considering the complexification.)

On several occasions we will use the following theorem due to Gelfand (see [37, Theorem 4.10.1]). If $T$ is an element of a complex unital Banach algebra $\mathfrak{A}$, for example, $\mathfrak{A}=\mathscr{L}(X)$, with $\sigma(T)=\{1\}$ and $\sup _{n \in \mathbb{Z}}\left\|T^{n}\right\|<\infty$, then $T=1$.

Since $\mathrm{GL}(X)$ is a norm-open subset of the Banach space $\mathscr{L}(X)$, it is a BanachLie group, but is of course far from being a (finite-dimensional) Lie group. As with any Banach-Lie group, $\mathrm{GL}(X)$ is NSS (has no small subgroups) in the norm topology. More precisely, we have the following.

\section{THEOREM 3.3}

Let $X$ be a Banach space. Then, in the norm topology, $\mathrm{GL}(X)$ has no small subgroups, that is, there is $\epsilon>0$ (in fact $\epsilon=\sqrt{2}$ ) such that

$$
\{T \in \mathrm{GL}(X) \mid\|T-\mathrm{Id}\|<\epsilon\}
$$

contains no nontrivial subgroup.

If follows that if $G \leq \mathrm{GL}(X)$ is locally compact, second countable in the norm topology, then $G$ is a Lie group.

\section{Proof}

Assume first that $X$ is a complex space. We claim that for any $T \in \mathrm{GL}(X)$ such that $\left(T^{n}\right)_{n \in \mathbb{Z}}$ is bounded, any $\lambda \in \sigma(T)$ is an approximate eigenvalue, that is, $T x_{n}-$ $\lambda x_{n} \rightarrow 0$ for some $x_{n} \in S_{X}$. For otherwise, $T-\lambda$ Id is bounded away from zero and hence will be an embedding of $X$ into $X$ whose range is a closed proper subspace of 
$X$. Since $X$ can be renormed so that $T$ is an isometry, we have that $\sigma(T) \subseteq \mathbb{T}$, and so we may find $\lambda_{n} \notin \sigma(T)$ such that $\lambda_{n} \rightarrow \lambda$. Therefore, if we choose $y \notin \operatorname{im}(T-\lambda \mathrm{Id})$, there are $x_{n} \in X$ such that $T x_{n}-\lambda_{n} x_{n}=y$ and so either $\left\|x_{n}\right\|$ is bounded or can be assumed to tend to infinity. In the second case, we see that for $z_{n}=\frac{x_{n}}{\left\|x_{n}\right\|}$ one has $T z_{n}-\lambda_{n} z_{n}=\frac{y}{\left\|x_{n}\right\|} \rightarrow 0$ and so also $T z_{n}-\lambda z_{n} \rightarrow 0$, contradicting that $T-\lambda$ Id is bounded away from 0 . And, in the first case,

$$
\left\|\left(T x_{n}-\lambda x_{n}\right)-y\right\|=\left\|\left(T x_{n}-\lambda x_{n}\right)-\left(T x_{n}-\lambda_{n} x_{n}\right)\right\|=\left|\lambda_{n}-\lambda\right| \cdot\left\|x_{n}\right\| \rightarrow 0,
$$

contradicting that $y$ is not in the closed subspace $\operatorname{im}(T-\lambda \mathrm{Id})$.

Now let $T \in \operatorname{GL}(X)$ satisfy $\left\|T^{n}-\operatorname{Id}\right\|<\sqrt{2}$ for all $n \in \mathbb{Z}$. If $\lambda \in \sigma(T)$, then $\lambda^{n}$ is an approximate eigenvalue of $T^{n}$ for any $n \in \mathbb{N}$, and so it follows that $\left|\lambda^{n}-1\right|<$ $\sqrt{2}$ for all $n \in \mathbb{N}$ and therefore that $\lambda=1$. So $\sigma(T)=\{1\}$. It suffices now to apply Gelfand's theorem to conclude that $T=\mathrm{Id}$. We have thus shown that $\{T \in \operatorname{GL}(X) \mid$ $\|T-\operatorname{Id}\|<\sqrt{2}\}$ contains no nontrivial subgroup.

If $X$ is a real Banach space, it suffices again to consider the complexification of $X$.

For the second part of the theorem, we note that by the Gleason-MontgomeryZippin-Yamabe solution to Hilbert's fifth problem (see, e.g., [40] for an exposition), any locally compact, second countable group with no small subgroups is a Lie group.

\subsection{Ideals and subgroups of $\mathrm{GL}(X)$}

Note that when $d \subseteq \mathscr{L}(X)$ is a two-sided operator ideal, the subgroup $\mathrm{GL}_{d}(X)$ of $\mathrm{GL}(X)$ consisting of all $\mathcal{\ell}$-perturbations of the identity, that is, invertible operators of the form

$$
T=\mathrm{Id}+A,
$$

where $A \in \mathcal{d}$, is normal in $\operatorname{GL}(X)$. Moreover, if $d$ is norm closed in $\mathscr{L}(X)$, then $\mathrm{GL}_{\ell}(X)$ is a norm-closed subgroup of $\mathrm{GL}(X)$.

Of particular importance for our investigation are the ideals of respectively finiterank, almost finite-rank, compact, strictly singular, and inessential operators. Namely,

- $\quad \mathcal{F}(X)=\{T \in \mathscr{L}(X) \mid T$ has finite rank $\}$;

- $\quad A \mathcal{F}(X)=\overline{\mathcal{F}(X)}\|\cdot\|$

- $\quad \mathcal{K}(X)=\{T \in \mathscr{L}(X) \mid T$ is compact $\}$

- $\quad \delta(X)=\{T \in \mathscr{L}(X) \mid T$ is strictly singular $\}$

- $\quad \ln (X)=\{T \in \mathscr{L}(X) \mid T$ is inessential $\}$.

Here an operator $T \in \mathscr{L}(X)$ is said to be strictly singular if there is no infinitedimensional subspace $Y \subseteq X$ such that $T: Y \rightarrow X$ is an isomorphic embedding. Also, $T \in \mathscr{L}(X)$ is inessential if for any $S \in \mathscr{L}(X)$, the operator Id $+S T$ is Fredholm, 
that is, has closed image, finite-dimensional kernel, and finite corank. In particular, for any $T \in \ln (X)$ and $t \in[0,1], \mathrm{Id}+t T$ is Fredholm and $\mathrm{Id}+T$ must have Fredholm index 0 , since the index is norm continuous and (Id $+t T)_{t \in[0,1]}$ is a continuous path from Id to Id $+T$. (More information about the ideal of inessential operators may be found in [32].) We then have the following inclusions,

$$
\mathcal{F}(X) \subseteq \mathcal{A} \mathcal{F}(X) \subseteq \mathcal{K}(X) \subseteq \&(X) \subseteq \ln (X)
$$

which give us similar inclusions between the corresponding subgroups of $\operatorname{GL}(X)$ that, for simplicity, we denote respectively by

$$
\mathrm{GL}_{f}(X) \subseteq \mathrm{GL}_{a f}(X) \subseteq \mathrm{GL}_{c}(X) \subseteq \mathrm{GL}_{s}(X) \subseteq \mathrm{GL}_{i n}(X)
$$

We also note that the ideals $\mathscr{A} \mathcal{F}(X), \mathcal{K}(X)$, and $\mathscr{S}(X)$ are norm closed in $\mathscr{L}(X)$. The group $\mathrm{GL}_{c}(X)$ is usually called the Fredholm group, though sometimes this refers more specifically to $\mathrm{GL}_{c}\left(\ell_{2}\right)$.

To simplify notation, we also let

$$
\operatorname{Isom}_{f}(X)=\operatorname{Isom}(X) \cap \mathrm{GL}_{f}(X)
$$

and

$$
\operatorname{Isom}_{a f}(X)=\operatorname{Isom}(X) \cap \mathrm{GL}_{a f}(X)
$$

denote the normal subgroups of $\operatorname{Isom}(X)$ consisting of all so-called finite-dimensional (resp., almost finite-dimensional) isometries. By reason of Theorem 3.4 below, these are the only two cases we are interested in.

Note that, since the compact operators form the only nontrivial norm-closed ideal of $\mathscr{L}\left(\ell_{2}\right)$ (see [15]), we have

$$
\operatorname{GL}_{a f}\left(\ell_{2}\right)=\mathrm{GL}_{c}\left(\ell_{2}\right)=\mathrm{GL}_{s}\left(\ell_{2}\right) .
$$

Similarly, if $X$ has the approximation property, then $\mathcal{K}(X)=\mathcal{A} \mathcal{F}(X)$ and hence $\mathrm{GL}_{a f}(X)=\mathrm{GL}_{c}(X)$. Though these equalities do not hold for general Banach spaces, as we will see now, any bounded subgroup of $\mathrm{GL}_{i n}(X)$ is contained in $\mathrm{GL}_{a f}(X)$, so from our perspective, there is no loss of generality in only considering $\mathrm{GL}_{a f}(X)$. It should be noted that Theorem 3.4 generalizes and simplifies results of Räbiger and Ricker ([55, Theorem 3.5], [56, Proposition 2.2]) and Ferenczi and Galego [29, Proposition 49].

THEOREM 3.4

Let $X$ be a Banach space, and let $G \leq \mathrm{GL}_{i n}(X)$ be a bounded subgroup. Then $G$ is contained in $\mathrm{GL}_{a f}(X)$. 


\section{Proof}

It suffices to show that if $T \in \mathrm{GL}_{i n}(X)$ and $\left\{T^{n} \mid n \in \mathbb{Z}\right\}$ is bounded, then $T \in$ $\mathrm{GL}_{a f}(X)$. For this, we may assume that $X$ is infinite-dimensional.

Suppose first that $X$ is complex, and work in the norm topology on $\mathscr{L}(X)$. Consider the quotient algebra

$$
\mathscr{B}=\mathscr{L}(X) / \mathscr{A} \mathcal{F}(X),
$$

and let

$$
\alpha: \mathscr{L}(X) \rightarrow \mathscr{B}
$$

be the corresponding quotient map.

Fix $T=\operatorname{Id}+U \in \mathrm{GL}_{i n}(X)$, and note that if $\lambda \neq 1$, then $T-\lambda \mathrm{Id}=(1-\lambda)(\operatorname{Id}+$ $U /(1-\lambda))$ is Fredholm with index 0 , and so $T-\lambda$ Id is a perturbation of an invertible operator by an operator in $\mathcal{F}(X)$. Therefore, $\alpha(T-\lambda$ Id $)=\alpha(T)-\lambda \alpha($ Id $)$ is an invertible element of $\mathscr{B}$ and hence $\lambda \notin \sigma(\alpha(T))$. We deduce that $\sigma(\alpha(T))=\{1\}$, and since $\left\{\alpha(T)^{n} \mid n \in \mathbb{Z}\right\}$ is bounded in the unital Banach algebra $\mathscr{B}$, Gelfand's theorem implies that $\alpha(T)=\alpha(\mathrm{Id})$. So $T$ - Id belongs to $\mathcal{A} \mathscr{F}(X)$, which concludes the proof of the complex case. Note that our proof in fact applies to any ideal $\mathcal{U}$ containing the finite-rank operators and such that any $\mathcal{U}$-perturbation of Id is Fredholm.

If instead $X$ is real, we consider its complexification $\hat{X}$ and the ideal

$$
u=\ln (X)+i \ln (X)
$$

of $\mathscr{L}(\hat{X})$, and observe that it contains $\mathcal{F}(\hat{X})=\mathcal{F}(X)+i \mathcal{F}(X)$.

We claim that for all $U+i V$ in $U$, Id $+U+i V$ is Fredholm on $\hat{X}$. Admitting the claim, we see that given $T \in \mathrm{GL}_{i n}(X)$, one can apply the proof in the complex case to $\hat{T}$, which is a $U$-perturbation of Id, and since then $\hat{T} \in \mathrm{GL}_{a f}(\hat{X})$ deduce that $T \in \mathrm{GL}_{a f}(X)$, thereby concluding the proof.

To prove the claim, note that since $\mathrm{Id}+U$ is Fredholm with index 0 on $X$, there exist $A \in \operatorname{GL}(X)$ and $F \in \mathscr{F}(X)$ such that $\mathrm{Id}+U=A+F$. Then

$$
\mathrm{Id}+U+i V=A+F+i V=A\left(\mathrm{Id}+A^{-1} F+i A^{-1} V\right),
$$

which indicates that it is enough to prove that $\mathrm{Id}+i V$ is Fredholm for any $V \in \mathcal{U}$.

Fix such a $V$, write $\mathrm{Id}+V^{2}=B+L$, where $B \in \mathrm{GL}(X)$ and $L \in \mathcal{F}(X)$, let $F$ be the finite-dimensional subspace $B^{-1} L X$, let $H$ be a closed subspace such that $X=F \oplus H$, let $\delta=d\left(S_{H+i H}, F+i F\right)>0$, and let $\epsilon>0$ be such that $\sqrt{2} \epsilon\left\|B^{-1}\right\|(1+\|V\|)<\delta$.

Let $x, y \in X$, and assume that $\|(\operatorname{Id}+i V)(x+i y)\| \leq \epsilon$. An easy computation shows that 


$$
\|x-V y\| \leq \epsilon
$$

and

$$
\|V x+y\| \leq \epsilon,
$$

whereby

$$
\|B x+L x\|=\left\|\left(\operatorname{Id}+V^{2}\right) x\right\| \leq \epsilon(1+\|V\|),
$$

and

$$
d(x, F) \leq \epsilon\left\|B^{-1}\right\|(1+\|V\|)<\delta / \sqrt{2} .
$$

Similarly $d(y, F)<\delta / \sqrt{2}$, and so $d(x+i y, F+i F)<\delta$. Conversely, this means that if $x+i y$ is a norm 1 vector in $H+i H$, then

$$
\|(\mathrm{Id}+i V)(x+i y)\|>\epsilon,
$$

and so the restriction of $\mathrm{Id}+i V$ to the finite-codimensional subspace $H+i H$ is an isomorphism onto its image. This proves that $\mathrm{Id}+i V$ has finite-dimensional kernel and closed image. In particular, its Fredholm index is defined, with the possible value $-\infty$, but then the continuity of the index implies that this index is zero and therefore that $\mathrm{Id}+i V$ is Fredholm. This concludes the proof of the claim and of the theorem.

Note that if $\mathbb{K}$ denotes the scalar field of $X$, then

$$
\mathbb{K}^{\star}=\{\lambda \operatorname{Id} \mid \lambda \in \mathbb{K} \backslash\{0\}\}
$$

is a norm-closed subgroup of $\operatorname{GL}(X)$. Also, if $d$ is a proper ideal in $\mathscr{L}(X)$, then $\mathbb{K}^{\star} \cap \mathrm{GL}_{\ell}(X)=\{\mathrm{Id}\}$, and so the group

$$
\{\lambda \mathrm{Id}+A \in \mathrm{GL}(X) \mid \lambda \in \mathbb{K} \backslash\{0\} \text { and } A \in \mathcal{d}\}
$$

of nonzero scalar multiples of elements of $\mathrm{GL}_{\ell}(X)$ splits as a direct product

$$
\mathbb{K}^{\star} \times \mathrm{GL}_{d}(X) .
$$

Moreover, since then $\mathcal{g}=\bar{d}$ is a norm-closed proper ideal, both $\mathbb{K}^{\star}$ and $\mathrm{GL}_{\mathcal{g}}(X)$ are norm closed and so the decompositions $\mathbb{K}^{\star} \times \mathrm{GL}_{\mathcal{g}}(X)$, and hence also $\mathbb{K}^{\star} \times \mathrm{GL}_{\mathcal{l}}(X)$, are topological direct products with respect to the norm topology. In particular, this applies to the ideals $\mathcal{F}$ and $\mathcal{A} \mathcal{F}$. So whereas our ultimate interest lies in, for example, the group $\mathbb{K}^{\star} \times \mathrm{GL}_{f}(X)$, in many situations this splitting allows us to focus on only the nontrivial part, namely, $\mathrm{GL}_{f}(X)$. 
PROPOSITION 3.5

Suppose that $X$ is a Banach space with separable dual and that $\pi: G \rightarrow \mathbb{K}^{\star} \times$ $\mathrm{GL}_{i n}(X)$ is a bounded, weakly continuous representation of a Polish group $G$. Then $\pi$ is norm continuous.

It follows that if $G \leq \mathbb{K}^{\star} \times \mathrm{GL}_{i n}(X)$ is a bounded subgroup, strongly closed in $\mathrm{GL}(X)$, then the strong operator and norm topologies coincide on $G$.

Proof

Composing $\pi$ with the coordinate projection from $\mathbb{K}^{\star} \times \mathrm{GL}_{i n}(X)$ onto $\mathrm{GL}_{i n}(X)$ and using Theorem 3.4, we see that the representation has image in $\mathbb{K}^{\star} \times \mathrm{GL}_{a f}(X)$. We remark that, since $X^{*}$ is separable, the ideal $\mathcal{F}(X)$ of finite-rank operators on $X$ is separable for the norm topology, whence also $\mathcal{A} \mathcal{F}(X)=\overline{\mathcal{F}}(X)^{\|\cdot\|}$ and $\mathbb{K}^{\star} \times$ $\mathrm{GL}_{a f}(X)$ are norm separable. Moreover, by Proposition 3.1, $\pi$ is strongly continuous, whereby, for every $\epsilon>0$ and $x \in X$, the set

$$
u_{\epsilon, x}=\{g \in G \mid\|\pi(g) x-x\|<\epsilon\}
$$

is open in $G$. Thus, if $\left\{x_{n}\right\}_{n \in \mathbb{N}} \subseteq X$ is dense in the unit ball of $X$, we see that

$$
\{g \in G \mid\|\pi(g)-\operatorname{Id}\|<\epsilon\}=\bigcup_{m \geq 1} \bigcap_{n \in \mathbb{N}} u_{\epsilon-1 / m, x_{n}}
$$

is Borel in $G$. So $\pi$ is a Borel-measurable homomorphism from a Polish group to a norm-separable topological group and therefore is norm continuous by Pettis's theorem (see [41, (9.10)]).

If now instead $G \leq \mathbb{K}^{\star} \times \mathrm{GL}_{i n}(X)$ is a bounded subgroup, strongly closed in $\operatorname{GL}(X)$, then $G$ is Polish in the strong operator topology and so the tautological representation on $X$ is norm continuous, implying that every norm-open set in $G$ is also strongly open. It follows that the two topologies coincide on $G$.

Observe that if a space $X$ has an unconditional basis and $G$ is the bounded group of isomorphisms acting by change of signs of the coordinates on the basis, then $G$ is an uncountable discrete group in the norm topology and is just the Cantor group $\prod_{n \in \mathbb{N}} \mathbb{Z}_{2}$ in the strong operator topology. So there is no hope of extending Proposition 3.5 to arbitrary strongly closed bounded subgroups $G \leq \mathrm{GL}(X)$ when $X$ has an unconditional basis, and, in many cases, to ensure the norm separability of any bounded $G \leq \mathrm{GL}(X)$, we even have to assume that $X$ does not contain any unconditional basic sequences.

\subsection{Near triviality}

As a corollary of Proposition 3.5 and Theorem 3.3, one sees that if $X$ is a Banach space with separable dual and $G \leq \mathrm{GL}_{i n}(X)$ is compact in the strong operator topol- 
ogy, then $G$ is a compact Lie group. However, we can prove an even stronger result that also allows us to bypass the result of Gleason-Montgomery-Zippin-Yamabe. The central notion here is that of near triviality.

\section{Definition 3.6}

Let $X$ be a Banach space, and let $G \leq \mathrm{GL}(X)$ be a subgroup. We say that $G$ acts nearly trivially on $X$ if $X$ admits a decomposition into $G$-invariant subspaces,

$$
X=H \oplus F
$$

such that $F$ is finite-dimensional and for all $T \in G$ there exists $\lambda_{T}$ such that $\left.T\right|_{H}=$ $\lambda_{T} \operatorname{Id}_{H}$.

We remark that a subgroup $G \leq \operatorname{GL}(X)$ acts nearly trivially on $X$ if and only if the subgroup $c(G)$ of GL $(\hat{X})$ acts nearly trivially on the complexification $\hat{X}$.

Note that, when $G$ acts nearly trivially on $X$, the strong operator topology on $G$ is just the topology of pointwise convergence on $F^{\prime}=F \oplus L$, where $L$ is an arbitrary one-dimensional subspace of $H$ (or $L=\{0\}$ if $H$ is trivial), and so $T \in$ $\left.G \mapsto T\right|_{F^{\prime}} \in \mathrm{GL}\left(F^{\prime}\right)$ is a topological group embedding. Since any strongly closed bounded subgroup of $\operatorname{GL}\left(F^{\prime}\right)$ is a compact Lie group, it follows that if $G$ is strongly closed and bounded in $\operatorname{GL}(X)$, then $G$ is also a compact Lie group.

We also remark that in this case one has $\operatorname{im}\left(T-\lambda_{T} \mathrm{Id}\right) \subseteq F$ for all $T \in G$. But, in fact, this observation leads to the following equivalent characterization of near triviality for bounded subgroups, which, for simplicity, we only state for subgroups of $\mathrm{GL}_{f}(X)$.

\section{LEMMA 3.7}

Let $X$ be a Banach space, and let $G \leq \mathrm{GL}_{f}(X)$ be a bounded subgroup. Then the following are equivalent:

(1) $G$ acts nearly trivially on $X$.

(2) There is a finite-dimensional $F \subseteq X$ such that $\operatorname{im}(T-\mathrm{Id}) \subseteq F$ for all $T \in G$. Moreover, in this case,

$$
X=\bigcap_{T \in G} \operatorname{ker}(T-\mathrm{Id}) \oplus \operatorname{span}\left(\bigcup_{T \in G} \operatorname{im}(T-\mathrm{Id})\right)
$$

is a decomposition witnessing near triviality.

\section{Proof}

One direction has already been noted, so suppose instead that (2) holds, and let $F \subseteq X$ be the finite-dimensional subspace $F=\operatorname{span}\left(\bigcup_{T \in G} \operatorname{im}(T-\mathrm{Id})\right)$. Then, for all $x \in X$ 
and $T \in G$, we have $T x=x+f$ for some $f \in F$ with $\|f\| \leq\|T-\operatorname{Id}\| \cdot\|x\|$. So, for any $x \in X, G \cdot x \subseteq x+(2\|G\|\|x\|) B_{F}$, showing that the orbit of $x$ is relatively compact. Moreover, $F$ is $G$-invariant, and if $Y \subseteq X$ is any $G$-invariant subspace with $Y \cap F=\{0\}$, then $T x=x$ for all $x \in Y$ and $T \in G$.

Since $G$ is almost periodic, by Proposition 3.2, $X$ is the closed linear span of its finite-dimensional irreducible subspaces. Therefore, as $Y \subseteq \bigcap_{T \in G} \operatorname{ker}(T-$ Id) for any irreducible $Y \subseteq X$ with $Y \cap F=\{0\}$, we see that $X=F \oplus \bigcap_{T \in G} \operatorname{ker}(T-\mathrm{Id})$, which finishes the proof.

As easy applications, we have the following lemmas.

LEMMA 3.8

Suppose that $X$ is a Banach space and that $G \leq \mathrm{GL}_{f}(X)$ is a finitely generated bounded subgroup. Then $G$ acts nearly trivially on $X$.

Proof

Let $G=\left\langle T_{1}, \ldots, T_{n}\right\rangle$, and put $F=\operatorname{im}\left(T_{1}-\mathrm{Id}\right)+\cdots+\operatorname{im}\left(T_{n}-\mathrm{Id}\right)$, which is finitedimensional. Note now that $T_{i}^{-1}-\mathrm{Id}=\left(T_{i}-\mathrm{Id}\right)\left(-T_{i}^{-1}\right)$, and $\operatorname{so} \operatorname{im}\left(T_{i}^{-1}-\mathrm{Id}\right)=$ $\operatorname{im}\left(T_{i}-\mathrm{Id}\right) \subseteq F$. Moreover, for $T, S \in \mathrm{GL}(X)$,

$$
T S-\text { Id }=(S-\text { Id })+(T-\text { Id }) S,
$$

and so, if $\operatorname{im}(T-$ Id $) \subseteq F$ and $\operatorname{im}(S-$ Id $) \subseteq F$, then also $\operatorname{im}(T S-$ Id $) \subseteq F$. It thus follows that $\operatorname{im}(T-$ Id) $\subseteq F$ for all $T \in G$, whence Lemma 3.7 applies.

LEMMA 3.9

Suppose that $X$ is a Banach space and that $T \in \mathrm{GL}_{f}(X)$ is an isometry. Then

$$
X=\operatorname{ker}(T-\mathrm{Id}) \oplus \operatorname{im}(T-\mathrm{Id}) .
$$

Moreover, if $X$ is complex, there are eigenvectors $x_{i}$ such that $\operatorname{im}(T-\mathrm{Id})=\left[x_{1}\right.$, $\left.\ldots, x_{n}\right]$.

\section{Proof}

As in Lemma 3.8, we see that $\operatorname{im}\left(T^{n}-\mathrm{Id}\right) \subseteq \operatorname{im}(T-\mathrm{Id})$ for all $n \in \mathbb{Z}$. The result now follows from Lemma 3.7. The "moreover" part follows from the fact that any isometry of a finite-dimensional complex space can be diagonalized.

PROPOSITION 3.10

Suppose that $X$ is a separable Banach space and that $G \leq \mathrm{GL}_{i n}(X)$ is a normcompact subgroup of $\mathrm{GL}(X)$. Then $G$ acts nearly trivially on $X$. 


\section{Proof}

There are several ways of proving this, for example, one based on the structure theory for norm-continuous representations (see [62]) of compact groups. But we will give a simple direct argument as follows.

Since $G$ is norm compact, it is almost periodic, so by Proposition 3.2, $X$ is the closed linear span of its finite-dimensional $G$-invariant subspaces. So, as $X$ is separable, by taking finite sums of these we can find an increasing sequence $F_{0} \subseteq F_{1} \subseteq$ $F_{2} \subseteq \cdots \subseteq X$ of finite-dimensional $G$-invariant subspaces such that $X=\overline{\bigcup_{n \geq 0} F_{n}}$.

Let $\mathfrak{A} \subseteq \mathscr{L}(X)$ be the subalgebra generated by $G$, and define, for every $n$, the unital algebra homomorphism $\pi_{n}: \mathfrak{A} \rightarrow \mathscr{L}\left(X / F_{n}\right)$ by

$$
\pi_{n}(A)\left(x+F_{n}\right)=A x+F_{n} .
$$

Note that $\left\|\pi_{n}(A)\right\| \leq\left\|\pi_{m}(A)\right\| \leq\|A\|$ for all $n \geq m$.

We claim that there is an $n$ such that $\pi_{n}(T-\mathrm{Id})=0$ for all $T \in G$. To see this, assume the contrary, and note that, by Theorem 3.3, for every $n$ there is some $T_{n} \in G$ such that $\left\|\pi_{n}\left(T_{n}-\mathrm{Id}\right)\right\|=\left\|\pi_{n}\left(T_{n}\right)-\mathrm{Id}\right\| \geq \sqrt{2}$. So for $m \leq n$, we have

$$
\left\|\pi_{m}\left(T_{n}-\mathrm{Id}\right)\right\| \geq\left\|\pi_{n}\left(T_{n}-\mathrm{Id}\right)\right\| \geq \sqrt{2} .
$$

Moreover, by passing to a subsequence, we can suppose that the $T_{n}$ converge in norm to some $T \in G$, whence $\left\|\pi_{m}(T-\mathrm{Id})\right\| \geq \sqrt{2}$ for all $m$.

Now, by Theorem 3.4, $G \leq \mathrm{GL}_{a f}(X)$, so $A=T-\mathrm{Id} \in \mathcal{A} \mathcal{F}$. Since $A$ is a norm limit of finite-rank operators, there is a finite-dimensional subspace $F \subseteq X$ such that $d(A x, F)<1$ for all $x \in X,\|x\| \leq 1$. Using that the $F_{n}$ are increasing and that $X=$ $\bigcup_{n \geq 0} F_{n}$, we see that there is an $n$ such that

$$
\sqrt{2} \leq\left\|\pi_{n}(A)\right\|=\sup _{\|x\|=1} d\left(A x, F_{n}\right) \leq \sup _{\|x\|=1} d(A x, F)+1 / 3 \leq 4 / 3,
$$

which is absurd. So fix an $n$ such that $\pi_{n}(T-\mathrm{Id})=0$ and thus $\operatorname{im}(T-\mathrm{Id}) \subseteq F_{n}$ for all $T \in G$. The result now follows from Lemma 3.7.

\section{THEOREM 3.11}

Suppose that $X$ is a Banach space with separable dual and that $G \leq \mathrm{GL}_{i n}(X)$ is an almost periodic subgroup, strongly closed in $\operatorname{GL}(X)$. Then $G$ acts nearly trivially on $X$ and hence is a compact Lie group.

\section{Proof}

By Proposition 3.2, $G=\bar{G}^{\text {SOT }}$ is compact in the strong operator topology. Since $X^{*}$ is separable, by Proposition 3.5 the norm and strong operator topologies coincide on $G$. Therefore, Proposition 3.10 applies. 
Recall that by Theorem 3.4, any isometry, which is an inessential perturbation of Id, must belong to $\operatorname{Isom}_{a f}(X)$. The next lemma shows that under additional conditions one may replace $\operatorname{Isom}_{a f}(X)$ by $\operatorname{Isom}_{f}(X)$.

LEMMA 3.12

Let $X$ be a complex Banach space, and let $T \in \operatorname{Isom}_{a f}(X)$. If $T$ has finite spectrum, then $T \in \operatorname{Isom}_{f}(X)$.

\section{Proof}

Write $\sigma(T)=\left\{1, \lambda_{1}, \ldots, \lambda_{n}\right\}$, and let $P$ be the spectral projection of $T$ corresponding to the spectral set $\{1\}$. Then $T(P X)=P X, \sigma\left(\left.T\right|_{P X}\right)=\{1\}$, and $\sup _{n \in \mathbb{Z}}\left\|\left(\left.T\right|_{P X}\right)^{n}\right\|<\infty$, so, by the result of Gelfand, $\left.T\right|_{P X}=$ Id. By the same reasoning $\left.T\right|_{X_{i}}=\lambda_{i} \operatorname{Id}_{X_{i}}$, where $X_{i}$ is the range of the spectral projection associated to $\left\{\lambda_{i}\right\}$ for $i=1, \ldots, n$. Since $T$ is an almost finite-rank perturbation of the identity, all elements of $\sigma(T)$ different from 1 have finite multiplicity and therefore $P X$ has finite codimension.

As in the case of $\mathrm{GL}_{f}(X)$, it will often be enough to study the group $\operatorname{Isom}_{f}(X)$, although our interest will really be in the subgroup $\{-1,1\} \times \operatorname{Isom}_{f}(X)$ of $\operatorname{Isom}(X)$ (resp., $\mathbb{T} \times \operatorname{Isom}_{f}(X)$ in the complex case). The same holds in relation to $\operatorname{Isom}_{a f}(X)$ and $\{-1,1\} \times \operatorname{Isom}_{a f}(X)$ (resp., $\mathbb{T} \times \operatorname{Isom}_{a f}(X)$ in the complex case).

\section{Decompositions of separable reflexive spaces by isometries}

\subsection{Duality mappings}

Let $X$ be a Banach space. Recall that a support functional for $x \in S_{X}$ is a functional $\phi$ in $S_{X *}$ such that $\phi(x)=1$. Support functionals always exist by the Hahn-Banach theorem. We denote by $J x$ the set of support functionals of $x \in S_{X}$ and extend $J$ to all of $X$ by positive homogeneity; that is, $J(t x)=t J x$ for all $t \geq 0$ and $x \in S_{X}$. Also, for $Y \subseteq X, J[Y]$ denotes the set of support functionals for $x \in Y$.

The following lemma finds its roots in Lindenstrauss's early work (see [43] or [21, Chapter VI]).

\section{LEMMA 4.1}

Let $X$ be a Banach space, and let $Y$ be a closed linear subspace of $X$. Then the following hold:

(a) $\quad Y$ and $J[Y]_{\perp}$ form a direct sum in $X$, and the corresponding projection from the subspace 


$$
Y \oplus J[Y]_{\perp}
$$

onto $Y$ has norm at most 1.

(b) If $Y$ is reflexive and $J[Y]$ is a closed linear subspace of $X^{*}$, then

$$
X^{*}=J[Y] \oplus Y^{\perp}
$$

and the corresponding projection from $X^{*}$ onto $J[Y]$ has norm at most 1 .

Proof

Suppose that $y \in S_{Y}, z \in J[Y]_{\perp}$, and let $\phi \in J y$. Then

$$
\|y+z\| \geq \phi(y+z)=\phi(y)=1=\|y\|,
$$

which implies (a). A similar argument shows that if $J[Y]$ is a closed linear subspace of $X^{*}$, then $J[Y]$ and $Y^{\perp}$ form a direct sum in $X^{*}$, and the corresponding projection from the subspace

$$
J[Y] \oplus Y^{\perp}
$$

onto $J[Y]$ has norm at most 1 .

For (b), assume that $Y$ is reflexive and that $J[Y]$ is a closed linear subspace of $X^{*}$. To see that $X^{*}=J[Y] \oplus Y^{\perp}$, fix $\psi \in X^{*}$, and let $\phi \in X^{*}$ be a Hahn-Banach extension of $\left.\psi\right|_{Y}$ to all of $X$ with $\|\phi\|=\left\|\left.\psi\right|_{Y}\right\|$. Then $\left.\psi\right|_{Y}=\left.\phi\right|_{Y}$, whence $\psi-\phi \epsilon$ $Y^{\perp}$ and $\|\phi\|=\left\|\left.\psi\right|_{Y}\right\|=\left\|\left.\phi\right|_{Y}\right\|$. On the other hand, since $\|\phi\|=\left\|\left.\phi\right|_{Y}\right\|$ and $Y$ is reflexive, $\left.\phi\right|_{Y}$ and thus $\phi$ attain their norm on $Y$, which means that $\phi=J y$ for some $y \in Y$, that is, $\phi \in J[Y]$. So $\psi=\phi+(\psi-\phi) \in J[Y] \oplus Y^{\perp}$.

We refer the reader to [21] for more general results in this direction (see, e.g., [21, Lemma 2.4, p. 239] for information about the class of weakly countably determined spaces).

Recall that a norm $\|\cdot\|$ on a Banach space $X$ is Gâteaux differentiable if, for all $x \in S_{X}$ and $h \in X$,

$$
\lim _{t \rightarrow 0, t \in \mathbb{R}} \frac{\|x+t h\|-\|x\|}{t}
$$

exists, in which case it is a continuous linear function in $h$. We note that this only depends on the $\mathbb{R}$-linear structure of $X$. When the norm on $X$ is Gateaux differentiable, the support functional is unique for all $x \in S_{X}$. This is proved in [21] in the real case and is also true in the complex case, as observed in Section 2.2. So, provided that the norm is Gâteaux differentiable, $J x$ is a singleton for all $x \in S_{X}$ and we can therefore see $J$ as a map from $X$ into $X^{*}$. 
Therefore, assuming that the norms on $X$ and $X^{*}$ are both Gâteaux differentiable, the duality map is defined from $X$ to $X^{*}$ and from $X^{*}$ to $X^{* *}$, where to avoid confusion we denote the second by $J_{*}: X^{*} \rightarrow X^{* *}$. The following two lemmas are now almost immediate from the definition of $J$ and $J_{*}$.

LEMMA 4.2

Let $X$ be a reflexive space with a Gâteaux differentiable norm, whose dual norm is Gâteaux differentiable. Then $J: X \rightarrow X^{*}$ is a bijection with inverse $J_{*}: X^{*} \rightarrow X$.

LEMMA 4.3

Let $X$ have a Gâteaux differentiable norm, and let $T$ be an isometry of $X$. Then the maps $J T^{-1}$ and $T^{*} J$ coincide on $X$.

Proof

For any $x \in S_{X}, T^{*}(J x)\left(T^{-1} x\right)=1$, which shows that $J\left(T^{-1} x\right)=T^{*}(J x)$. This extends to all of $X$ by positive homogeneity.

\subsection{LUR renormings and isometries}

Recall that a norm on a Banach space $X$ is uniformly convex if

$$
\forall \epsilon>0 \exists \delta>0 \forall x, y \in S_{X}(\|x-y\| \geq \epsilon \Rightarrow\|x+y\| \leq 2-\delta) .
$$

A weaker yet important notion in renorming theory is that of a locally uniformly rotund (LUR) norm:

$$
\forall x_{0} \in S_{X} \forall \epsilon>0 \exists \delta>0 \forall x \in S_{X}\left(\left\|x-x_{0}\right\| \geq \epsilon \Rightarrow\left\|x+x_{0}\right\| \leq 2-\delta\right) .
$$

(For other characterizations of LUR norms, we refer to [21].) Any LUR norm is strictly convex, meaning that the associated closed unit ball is strictly convex. Note that this definition does not depend on $X$ being seen as real or complex.

PROPOSITION 4.4

Assume that $X$ is a Banach space whose dual norm is LUR. Then the duality mapping $J: X \rightarrow X^{*}$ is well defined and norm continuous.

Therefore, if $X$ is reflexive and both the norm and the dual norm are LUR, then $J: X \rightarrow X^{*}$ is a norm homeomorphism with inverse $J_{*}: X^{*} \rightarrow X$.

\section{Proof}

It follows from the LUR property in $X^{*}$ that the norm on $X$ is Gâteaux differentiable (see [21]) and that therefore $J$ is well defined. Now given $x_{0} \in S_{X}$ and $\epsilon>0$, let $\delta>0$ be associated to $\epsilon$ by the LUR property at $\phi_{0}=J x_{0}$. If for some $x \in S_{X}$, 
$\left\|J x-J x_{0}\right\| \geq \epsilon$, then $\left\|J x+J x_{0}\right\| \leq 2-\delta$ and therefore

$$
\left|1-(J x)\left(x_{0}\right)\right|=\left|2-\left(J x+J x_{0}\right)\left(x_{0}\right)\right| \geq 2-\left\|J x+J x_{0}\right\| \geq \delta .
$$

Thus

$$
\left\|x-x_{0}\right\| \geq\left|(J x)\left(x-x_{0}\right)\right|=\left|1-(J x)\left(x_{0}\right)\right| \geq \delta .
$$

This proves that $J$ is continuous as a map from $S_{X}$ to $S_{X^{*}}$ and therefore from $X$ to $X^{*}$.

For a general characterization of LUR norms with duality mappings in possibly nonreflexive spaces, we refer the reader to the paper of Debs, Godefroy, and SaintRaymond [20].

While not all real separable spaces admit an equivalent uniformly convex norm, it is a well-known result of renorming theory, due to Kadec, that they admit an equivalent LUR norm (see [21, Chapter II]). However, we are interested in LUR renormings which, in some sense, keep track of the original group of isometries on the space. The next proposition is the first of a series of results in that direction.

For expositional ease, if $\|\cdot\|$ is a norm on a Banach space $X$, we will denote the induced norm on the dual space $X^{*}$ by $\|\cdot\|^{*}$.

\section{PROPOSITION 4.5}

Let $X$ be a Banach space, and let $G \leq \mathrm{GL}(X)$ be a bounded subgroup. Assume that $X$ admits $G$-invariant equivalent norms $\|\cdot\|_{0}$ and $\|\cdot\|_{1}$ such that $\|\cdot\|_{0}$ and $\|\cdot\|_{1}^{*}$ are $L U R$. Then $X$ admits a $G$-invariant equivalent norm $\|\cdot\|_{2}$ such that both $\|\cdot\|_{2}$ and $\|\cdot\|_{2}^{*}$ are $L U R$.

Moreover, for any $G$-invariant equivalent norm $\|\cdot\|$ on $X$ and $\epsilon>0$, one can choose $\|\cdot\|_{2}$ to be $(1+\epsilon)$-equivalent to $\|\cdot\|$.

\section{Proof}

The proof proceeds along the lines of [21, Section II.4], but we include some details for the convenience of the reader.

Let $\mathcal{N}$ denote the space of $G$-invariant equivalent norms on $X$ equipped with the complete metric

$$
d(\|\cdot\|,\|\cdot\|)=\sup _{x \neq 0} \mid \log \frac{\|x\|}{\|x\| \mid} .
$$

Let also $\mathscr{L} \subseteq \mathcal{N}$ denote the subset of all norms that are LUR. We first show that $\mathscr{L}$ is comeager in $\mathcal{N}$. 
Note first that $\|\cdot\|_{0} \in \mathscr{L}$, and define for all $k \geq 1$ the open set

$$
\mathscr{L}_{k}=\left\{\|\cdot\| \in \in \mathcal{N} \mid \exists\|\cdot\| \in \mathcal{N} \exists n \geq k d\left(\|\cdot\|,\left(\|\cdot\|^{2}+\frac{1}{n}\|\cdot\|_{0}^{2}\right)^{1 / 2}\right)<\frac{1}{n^{2}}\right\} .
$$

Now, if $\|\cdot\| \in \mathcal{N}$, then for all $n \geq k,\left(\|\cdot\|^{2}+\frac{1}{n}\|\cdot\|_{0}^{2}\right)^{1 / 2} \in \mathscr{L}_{k}$, while on the other hand $\left(\|\cdot\|^{2}+\frac{1}{n}\|\cdot\|_{0}^{2}\right)^{1 / 2} \underset{n \rightarrow \infty}{\longrightarrow}\|\cdot\|$, which shows that $\|\cdot\| \in \overline{\mathscr{L}_{k}}$ for every $k \geq 1$. Thus, $\mathscr{L}_{k}$ is dense open for every $k$ and hence $\bigcap_{k \geq 1} \mathscr{L}_{k}$ is comeager in $\mathcal{N}$ and, as shown in [21], is a subset of $\mathscr{L}$. So $\mathscr{L}$ is comeager in $\mathcal{N}$.

Similarly, one shows, using that $\|\cdot\|_{1}^{*}$ is LUR, that the set

$$
\mathcal{M}=\left\{\|\cdot\| \in \mathcal{N} \mid\|\cdot\|^{*} \text { is LUR }\right\}
$$

is comeager in $\mathcal{N}$. It thus suffices to choose $\|\cdot\|_{2}$ in the comeager and thus dense intersection $\mathscr{L} \cap \mathcal{M}$.

The following result was proved by Lancien (in [42], see Theorem 2.1 and Remark 1 on p. 639, and Theorem 2.3 and the observation and remark on p. 640).

THEOREM 4.6 (G. Lancien)

Let $(X,\|\cdot\|)$ be a separable Banach space, and set $G=\operatorname{Isom}(X,\|\cdot\|)$. Then the following hold.

(a) If $X$ has the Radon-Nikodym property, then $X$ admits an equivalent $G$-invariant LUR norm.

(b) If $X^{*}$ is separable, then $X$ admits an equivalent $G$-invariant norm whose dual norm is LUR.

We should note here that the result of Lancien is proved and stated for real spaces, but also holds for complex spaces. Indeed, suppose that $X$ is a complex space, and let $X_{\mathbb{R}}$ denote the space $X$ seen as a Banach space over the real field. Any equivalent real norm on $X_{\mathbb{R}}$, which is $\operatorname{Isom}\left(X_{\mathbb{R}},\|\cdot\|\right)$-invariant, must be invariant under all isometries of the form $\lambda \operatorname{Id}$ for $\lambda \in \mathbb{T}$ and hence is actually a complex norm that is $\operatorname{Isom}(X,\|\cdot\|)$ invariant. Thus, in order to obtain Lancien's result for a complex space $X$, and modulo the observations in Section 2.2, it suffices to simply apply it to $X_{\mathbb{R}}$.

Combining Proposition 4.5 and Theorem 4.6, plus the fact that any bounded subgroup of $\mathrm{GL}(X)$ is a group of isometries in some equivalent norm, we obtain the following result.

THEOREM 4.7

Let $X$ be a Banach space with the Radon-Nikodym property and separable dual, and let $G \leq \mathrm{GL}(X)$ be a bounded subgroup. Then $X$ admits an equivalent $G$-invariant LUR norm whose dual norm is also LUR. 
Recall that a Banach space $X$ is said to be super-reflexive if whenever $Y$ is a Banach space crudely finitely representable in $X$ (i.e., for some constant $K$, any finitedimensional subspace of $Y$ embeds with constant $K$ in $X)$, then $Y$ is reflexive. Moreover, $(X,\|\cdot\|)$ is super-reflexive if and only if it admits a uniformly convex renorming $(X,\|\cdot\|)$, and, in this case (see, e.g., [6, Proposition 2.3]), if $G=\operatorname{Isom}(X,\|\cdot\|)$, then

$$
\|x \mid\|_{0}=\sup _{T \in G}\|T x\|
$$

is a $G$-invariant uniformly convex renorming of $X$. This strengthens Theorem 4.7 in the case of super-reflexive spaces.

Since any reflexive space has the RNP (see [10]), the conclusion of Theorem 4.7 holds, in particular, for any separable reflexive space. As a first consequence of this, we have the following lemma (the existence of the fixed point can alternatively be obtained directly via Ryll-Nardzewski's fixed-point theorem [59]; see also [26, Theorem 12.22]).

LEMMA 4.8

Let $X$ be a separable reflexive space, and let $G$ be a bounded subgroup of $\mathrm{GL}(X)$. Then, for each $x \in X, \overline{\operatorname{conv}}(G \cdot x)$ contains a unique $G$-fixed point.

\section{Proof}

By renorming using Theorem 4.7, we may assume that $G$ is a group of isometries and that the norms on $X$ and $X^{*}$ are LUR. Fix $x \in X$, and let $C=\overline{\operatorname{conv}}(G \cdot x)$. By reflexivity, $C$ is weakly compact and thus contains a point $x_{0}$ of minimal norm. Furthermore, by strict convexity, $x_{0}$ is unique and therefore fixed by $G$.

Assume that $x_{1}$ is another $G$-fixed point in $C$, and let $\phi \in X^{*}$. As above, we may find a unique functional $\phi_{0}$ of minimal norm in $\overline{\operatorname{conv}}(G \cdot \phi)$, which is therefore fixed by $G$. It follows that $\phi_{0}$ is constant on $G \cdot x$ and thus also on $C=\overline{\operatorname{conv}}(G \cdot x)$, whence $\phi_{0}\left(x_{1}\right)=\phi_{0}\left(x_{0}\right)=\phi_{0}(x)$ and $\phi_{0}\left(x_{1}-x_{0}\right)=0$. Now, since $x_{1}-x_{0}$ is $G$-fixed, seen as a functional on $X^{*}$, it must be constant on $\overline{\operatorname{conv}}(G \cdot \phi)$ and so $\phi\left(x_{1}-x_{0}\right)=0$. As $\phi$ was arbitrary, this proves that $x_{1}=x_{0}$.

\subsection{Jacobs-de Leeuw-Glicksberg and Alaoglu-Birkhoff decompositions}

If $T$ is an operator on a Banach space $X$, we define the $T$-invariant (resp., $T^{*}$-invariant) subspaces:

- $\quad H_{T}=\operatorname{ker}(T-\mathrm{Id})$;

- $H_{T^{*}}=\operatorname{ker}\left(T^{*}-\mathrm{Id}\right)$;

- $\quad F_{T}=\operatorname{im}(T-\mathrm{Id})$;

- $\quad F_{T^{*}}=\operatorname{im}\left(T^{*}-\mathrm{Id}\right)$.

Then, as is easy to verify, $H_{T^{*}}=\left(F_{T}\right)^{\perp}, H_{T}=\left(F_{T^{*}}\right)_{\perp}$ and $\overline{F_{T}}=\left(H_{T^{*}}\right)_{\perp}$. 
Also, if $G \leq \mathrm{GL}(X)$ is a bounded group of automorphisms of $X$, we define the following closed $G$-invariant subspaces of $X$ and $X^{*}$ :

- $H_{G}=\{x \in X \mid x$ is fixed by $G\}$;

- $H_{G^{*}}=\left\{\phi \in X^{*} \mid \phi\right.$ is fixed by $\left.G\right\}$;

- $K_{G}=\{x \in X \mid x$ is almost periodic $\}$

- $K_{G^{*}}=\left\{\phi \in X^{*} \mid \phi\right.$ is almost periodic $\}$.

(Note that $H_{G} \subseteq K_{G}$ and $H_{G^{*}} \subseteq K_{G^{*}}$.) When $G$ is generated by a single element, that is, $G=\langle T\rangle$, obviously $H_{\langle T\rangle}, H_{\langle T\rangle^{*}}$ coincide with $H_{T}, H_{T^{*}}$.

Jacobs [38], and later de Leeuw and Glicksberg [19], studied canonical decompositions induced by weakly almost periodic semigroups $\mathbb{S} \subseteq \mathscr{L}(X)$ of operators on a Banach space $X$. As part of the subsequent development of their theory, the following result has been proved (see [11, Corollary 6.2.19] or Troallic [66] along with [19, Theorem 4.11], for proofs based on the Ryll-Nardzewski fixed-point theorem, resp., on Namioka's joint continuity theorem). If $X$ is a Banach space and $G$ is a weakly almost periodic subgroup of $\mathscr{L}(X)$, that is, every $G$-orbit is relatively weakly compact, then $X=K_{G} \oplus Z$, where $Z$ is a canonical complement consisting of the so-called furtive vectors, that is, vectors $x \in X$ so that $0 \in \overline{G \cdot x}^{\text {w }}$. We note that, whereas the set of furtive vectors does not a priori form a linear subspace of $X$, it is a closed linear subspace in the above case. Observe also that if $X^{*}$ is separable, then $x \in X$ is furtive if and only if there is a sequence of $T_{n} \in G$ so that $T_{n} x \underset{\mathrm{w}}{\longrightarrow} 0$.

We now instead give a new proof of this decomposition for separable reflexive $X$, using only the renorming given by Theorem 4.7 and the properties of the duality mapping. In fact, our proof identifies further decompositions of $X$ than those given by the approach of de Leeuw and Glicksberg and also equates $Z$ with $\left(K_{G^{*}}\right)_{\perp}$.

\section{LEMMA 4.9}

Let $X$ be a Banach space, and let $G \leq \mathrm{GL}(X)$ be a bounded subgroup. Then the following implications hold:

$$
\begin{array}{ccc}
x \text { is furtive } & \Rightarrow & x \in\left(K_{G^{*}}\right)_{\perp} \\
& \Downarrow \\
0 \in \frac{\Downarrow}{\operatorname{conv}}(G \cdot x) & \Rightarrow & x \in\left(H_{G^{*}}\right)_{\perp}
\end{array}
$$

\section{Proof}

If $x \in X$ is furtive and $\phi \in K_{G^{*}}$, we claim that $\phi(x)=0$. To see this, assume without loss of generality that $G$ is a group of isometries, fix $\epsilon>0$, and pick an $\epsilon$-dense subset $\psi_{1}, \ldots, \psi_{k}$ of $G \cdot \phi$. Then, since $x$ is furtive, there is some $T \in G$ such that $\left|T^{*} \psi_{i}(x)\right|=\left|\psi_{i}(T x)\right|<\epsilon$ for every $i=1, \ldots, k$. Picking $i$ such that $\left\|\phi-T^{*} \psi_{i}\right\|=$ $\left\|\left(T^{-1}\right)^{*} \phi-\psi_{i}\right\|<\epsilon$, we see that $|\phi(x)| \leq\left\|\phi-T^{*} \psi_{i}\right\|\|x\|+\left|T^{*} \psi_{i}(x)\right|<\epsilon\|x\|+\epsilon$. 
So, as $\epsilon>0$ is arbitrary, it follows that $\phi(x)=0$. In other words, any furtive vector belongs to $\left(K_{G^{*}}\right)_{\perp}$.

Note also that if $\phi \in H_{G^{*}}, T_{1}, \ldots, T_{n} \in G$ and $\lambda_{i} \geq 0$ are such that $\sum_{i=1}^{n} \lambda_{i}=1$, then, for any $x \in X$,

$$
\phi\left(\sum_{i=1}^{n} \lambda_{i} T_{i} x\right)=\sum_{i=1}^{n} \lambda_{i} T_{i}^{*} \phi(x)=\sum_{i=1}^{n} \lambda_{i} \phi(x)=\phi(x) .
$$

Hence, if $0 \in \overline{\operatorname{conv}}(G \cdot x)$ and $\phi \in H_{G^{*}}$, then $\phi(x)=0$, showing the second implication. Finally, the vertical implications are trivial.

It turns out that if $X$ is separable reflexive, then the horizontal implications reverse, which allows us to identify the Jacobs-de Leeuw-Glicksberg decomposition with a decomposition provided by the duality mapping. In this setting, we also obtain the decomposition originating in the work of Alaoglu and Birkhoff [1].

THEOREM 4.10

Let $X$ be a separable reflexive space, and suppose that $G \leq \mathrm{GL}(X)$ is a bounded subgroup. Then $X$ admits the following $G$-invariant decompositions.

(a) (Alaoglu-Birkhoff-type decomposition)

$$
X=H_{G} \oplus\left(H_{G^{*}}\right)_{\perp},
$$

where, if $\delta \subseteq G$ generates a dense subgroup of $G$, then

$$
\left(H_{G^{*}}\right)_{\perp}=\overline{\operatorname{span}}\left(\bigcup_{T \in S} F_{T}\right)=\{x \in X \mid 0 \in \overline{\operatorname{conv}}(G \cdot x)\} .
$$

Moreover, the projection $P: X \rightarrow H_{G}$ is given by

$$
P x=\text { the unique point in } H_{G} \cap \overline{\operatorname{conv}}(G \cdot x) \text {. }
$$

(b) (Jacobs-de Leeuw-Glicksberg-type decomposition)

$$
X=K_{G} \oplus\left(K_{G^{*}}\right)_{\perp},
$$

where

$$
\left(K_{G^{*}}\right)_{\perp}=\{x \in X \mid x \text { is furtive }\}=\left\{x \in X \mid \exists T_{n} \in G T_{n} x \underset{w}{\longrightarrow} 0\right\} .
$$

Moreover, the projections onto each summand have norm bounded by $2\|G\|^{2}$ (or $\|G\|^{2}$ when the summand is $H_{G}$ or $\left.K_{G}\right)$, and either $G$ is almost periodic, that is, $X=K_{G}$, or the subspace $\left(K_{G^{*}}\right)_{\perp}$ is infinite-dimensional. 
In particular, for any isometry $T$ of $X$,

$$
X=H_{T} \oplus \overline{F_{T}} .
$$

Proof

Renorming $X$ by $\|x\|\left\|=\sup _{T \in G}\right\| T x \|$, we can suppose that $G$ is a group of isometries. Moreover, by a further renorming using Theorem 4.7, we can suppose that both the norm and its dual are LUR. Furthermore, using the quantitative estimate of Proposition 4.5, and fixing $\epsilon>0$, we can ensure that the resulting norm on $X$ is $(1+\epsilon)\|G\|-$ equivalent to the original norm.

Note then that, by Propositions 4.4 and 4.3, $J: X \rightarrow X^{*}$ is a homeomorphism satisfying $J T^{-1}=T^{*} J$, whence $J\left[H_{G}\right] \subseteq H_{G^{*}}$ and $J\left[K_{G}\right] \subseteq K_{G^{*}}$. Similarly, the inverse $J_{*}$ satisfies $J_{*}\left[H_{G^{*}}\right] \subseteq H_{G}$ and $J_{*}\left[K_{G^{*}}\right] \subseteq K_{G}$, whence $J\left[H_{G}\right]=H_{G^{*}}$ and $J\left[K_{G}\right]=K_{G^{*}}$. It follows from Lemma 4.1(b) that

$$
X=H_{G} \oplus\left(H_{G^{*}}\right)_{\perp}=K_{G} \oplus\left(K_{G^{*}}\right)_{\perp},
$$

where the corresponding projections have norm at most 2, or 1 for the projections onto $H_{G}$ and $K_{G}$. Since $H_{G} \subseteq K_{G}$ and $H_{G^{*}} \subseteq K_{G^{*}}$, we see that the decompositions refine to $X=H_{G} \oplus\left(\left(H_{G^{*}}\right)_{\perp} \cap K_{G}\right) \oplus\left(K_{G^{*}}\right)_{\perp}$. Since $\epsilon$ was arbitrary, the estimate on the norms of the projections in the original space follows immediately.

Note that, since no nonzero $G$-orbit on $\left(K_{G^{*}}\right)_{\perp}$ is relatively compact, the latter space must either be infinite-dimensional or reduce to $\{0\}$.

Moreover, if $\delta \subseteq G$ generates a dense subgroup of $G$, then

$$
H_{G^{*}}=\bigcap_{T \in \mathcal{S}} H_{T^{*}}=\bigcap_{T \in \mathcal{S}}\left(F_{T}\right)^{\perp}=\left(\operatorname{span}\left(\bigcup_{T \in \mathcal{S}} F_{T}\right)\right)^{\perp}
$$

and so $\left(H_{G^{*}}\right)_{\perp}=\overline{\operatorname{span}}\left(\bigcup_{T \in \mathcal{S}} F_{T}\right)$.

Now, to see that $\left(K_{G^{*}}\right)_{\perp}$ is the set $Z$ of furtive vectors, note that, by the abovementioned Corollary 6.2.19 in [11], $Z$ is a closed linear subspace such that $X=$ $K_{G} \oplus Z$. As also $Z \subseteq\left(K_{G^{*}}\right)_{\perp}$, we have $Z=\left(K_{G^{*}}\right)_{\perp}$.

Observe now that for any $z \in\left(H_{G^{*}}\right)_{\perp}$, we have $\overline{\operatorname{conv}}(G \cdot z) \subseteq\left(H_{G^{*}}\right)_{\perp}$, and so the unique point in $H_{G} \cap \overline{\operatorname{conv}}(G \cdot z)$, that exists by Lemma 4.8, must be zero. Thus, if $y \in H_{G}$ and $z \in\left(H_{G^{*}}\right)_{\perp}$, then for any $\epsilon>0$ there are $T_{i} \in G$ and $\lambda_{i} \geq 1$ with $\sum_{i=1}^{n} \lambda_{i}=1$ and

$$
\left\|y-\sum_{i=1}^{n} \lambda_{i} T_{i}(y+z)\right\|=\left\|y-\left(\sum_{i=1}^{n} \lambda_{i} y+\sum_{i=1}^{n} \lambda_{i} T_{i} z\right)\right\|=\left\|\sum_{i=1}^{n} \lambda_{i} T_{i} z\right\|<\epsilon .
$$

Since $\epsilon>0$ is arbitrary, this shows that the projection of $x=y+z$ onto $H_{G}$, namely $y$, belongs to $H_{G} \cap \overline{\operatorname{conv}}(G \cdot x)$, which, by Lemma 4.8, implies that the projection $P$ 
of $X$ onto $H_{G}$ is given by $\{P x\}=H_{G} \cap \overline{\operatorname{conv}}(G \cdot x)$ and hence that $\left(H_{G^{*}}\right)_{\perp}=\{x \in$ $X \mid 0 \in \overline{\operatorname{conv}}(G \cdot x)\}$.

The above approach also provides other decompositions not identified in the Jacobs-de Leeuw-Glicksberg theory. Here, if $G$ is a bounded subgroup of GL( $X)$ and $\delta \subseteq G$ is a symmetric subset (thus not necessarily closed under composition), we let

- $K_{8}=\{x \in X \mid \& \cdot x$ is totally bounded in $X\}$,

- $K_{8^{*}}=\left\{\phi \in X^{*} \mid \boldsymbol{8} \cdot \phi\right.$ is totally bounded in $\left.X^{*}\right\}$,

and we note that this agrees with the previous definitions in the case $\delta=G$. A simple argument shows that $K_{\delta}$ and $K_{\delta^{*}}$ are closed linear subspaces of $X$ and $X^{*}$, respectively, though not necessarily $G$-invariant. Similarly, a vector $x \in X$ is a said to be 8 -furtive if $0 \in \overline{\delta \cdot x}^{\mathrm{w}}$. As in Lemma 4.9 , we see that every $\delta$-furtive vector belongs to $\left(K_{\mathcal{S}^{*}}\right)_{\perp}$. Moreover, the proof of Theorem 4.10 easily adapts to provide the following.

\section{PROPOSITION 4.11}

Let $X$ be a separable reflexive Banach space, let $G \leq \mathrm{GL}(X)$ be a bounded subgroup, and let $\delta \subseteq G$ be a symmetric subset. Then

$$
X=K_{8} \oplus\left(K_{8 *}\right)_{\perp},
$$

where the corresponding projection onto $K_{\S}$ has norm at most $\|G\|^{2}$.

\subsection{Finite-dimensional decompositions}

Theorem 4.10 has several applications in ergodic theory to the decompositions of functions over dynamical systems. We will now see how it can also provide the existence of finite-dimensional decompositions and even Schauder bases for Banach spaces.

For the following lemma, recall that an isometry $T$ of a Banach space $X$ is finitedimensional if it is a finite-rank perturbation of the identity on $X$. Recall also that a Schauder basis is always assumed to be infinite and thus it must be a basis for an infinite-dimensional space. Abusing terminology, we say that a Banach space $Y$ has a possibly finite Schauder basis if either $\operatorname{dim} Y<\infty$ or otherwise $\operatorname{dim} Y=\infty$ and $Y$ has a Schauder basis.

LEMMA 4.12

Let $X$ be a separable reflexive Banach space, and let $T_{1}, T_{2}, \ldots$ be a sequence of finite-dimensional isometries of $X$. Then the complemented subspace $\overline{\operatorname{span}}\left(\bigcup_{n \geq 1} F_{T_{n}}\right)$ admits a finite-dimensional decomposition with constant at most 2. 
If, moreover, the dimensions of $F_{T_{n}}$ are uniformly bounded, then $\overline{\operatorname{span}}\left(\bigcup_{n \geq 1} F_{T_{n}}\right)$ has a (possibly finite) Schauder basis.

\section{Proof}

By Theorem 4.10, we see that, for all $n \geq 1$,

$$
X=\left(F_{T_{1}}+\cdots+F_{T_{n}}\right) \oplus H_{\left\langle T_{1}, \ldots, T_{n}\right\rangle},
$$

where the corresponding projections $P_{n}$ of $X$ onto the summands $\left(F_{T_{1}}+\cdots+F_{T_{n}}\right)$ have norm uniformly bounded by 2 . Similarly,

$$
X=\overline{\operatorname{span}}\left(\bigcup_{n \geq 1} F_{T_{n}}\right) \oplus H_{\left\langle T_{1}, T_{2}, \ldots\right\rangle} .
$$

Also, for $n \leq m$, we have $\left(F_{T_{1}}+\cdots+F_{T_{n}}\right) \subseteq\left(F_{T_{1}}+\cdots+F_{T_{m}}\right)$ and $H_{\left\langle T_{1}, \ldots, T_{n}\right\rangle} \supseteq$ $H_{\left\langle T_{1}, \ldots, T_{m}\right\rangle}$, from which it follows that $P_{n} P_{m}=P_{m} P_{n}=P_{n}$. Clearly, $\| P_{n} x-$ $x \| \underset{n \rightarrow \infty}{\longrightarrow} 0$ for all $x \in \operatorname{span}\left(\bigcup_{n \geq 1} F_{T_{n}}\right)$ and so, as the $P_{n}$ are uniformly bounded, this holds for all $x \in \overline{\operatorname{span}}\left(\bigcup_{n \geq 1} F_{T_{n}}\right)$. By [44, p. 47], it follows that the finite-dimensional subspaces $E_{n}=\left(P_{n}-P_{n-1}\right)[X]$ form a finite-dimensional decomposition of the complemented closed subspace $\overline{\operatorname{span}}\left(\bigcup_{n \geq 1} F_{T_{n}}\right)$.

If the dimensions of the $F_{T_{n}}$ are uniformly bounded, then the dimensions of the $E_{n}$ are also uniformly bounded. In this case, we can further refine the decomposition to a Schauder basis for $\overline{\operatorname{span}}\left(\bigcup_{n \geq 1} F_{T_{n}}\right)$ provided of course that the latter is infinite-dimensional.

With Lemma 4.12 in hand, we can now prove several results on finite-dimensional decompositions.

\section{THEOREM 4.13}

Let $X$ be a separable reflexive Banach space, and let $G \leq \mathrm{GL}(X)$ be a bounded subgroup containing a nontrivial finite-rank perturbation of the identity. Then $X$ admits a $G$-invariant decomposition $X=Y \oplus Z$ with $Y \neq\{0\}$ and where $Y$ has a (possibly finite) Schauder basis.

\section{Proof}

Fix some $\mathrm{Id} \neq T \in G$ such that $F_{T}=\operatorname{im}(T-\mathrm{Id})$ is a finite-dimensional subspace of $X$, and renorm so that $G$ is a group of isometries. Since $G$ is separable in the strong operator topology, we can let $R_{1}, R_{2}, R_{3}, \ldots$ list the elements of a dense subgroup $\Gamma$ of $G$ and set $T_{n}=R_{n} T R_{n}^{-1}$, whereby $F_{T_{n}}=F_{R_{n} T R_{n}^{-1}}=R_{n}\left[F_{T}\right]$ and $H_{T_{n}}=$ $R_{n}\left[H_{T}\right]$. 
Now, by Theorem 4.10,

$$
X=\overline{\operatorname{span}}\left(\bigcup_{n \geq 1} F_{T_{n}}\right) \oplus H_{\left\langle T_{1}, T_{2}, \ldots\right\rangle} .
$$

Moreover, as $\Gamma=\left\{R_{1}, R_{2}, \ldots\right\}$, we see that both summands

$$
Y=\overline{\operatorname{span}}\left(\bigcup_{n \geq 1} F_{T_{n}}\right)=\overline{\operatorname{span}}\left(\bigcup_{n \geq 1} R_{n}\left[F_{T}\right]\right) \neq\{0\}
$$

and

$$
Z=H_{\left\langle T_{1}, T_{2}, \ldots\right\rangle}=\bigcap_{n \geq 1} R_{n}\left[H_{T}\right]
$$

are $\Gamma$-invariant and therefore also $G$-invariant.

Finally, applying Lemma 4.12 to the sequence $T_{n}$, we see that $Y$ has a (possibly finite) Schauder basis.

Note that if in Theorem 4.13 we have $K_{G}=\{0\}$, that is, no nonzero orbit is totally bounded, then there are no finite-dimensional $G$-invariant subspaces of $X$ and so $\operatorname{dim} Y=\infty$, whereby $Y$ has a Schauder basis. Observe also that the projection onto $Z$ has norm at most $\|G\|^{2}$ and that $F_{T} \subseteq Y$, where $T \in G$ is the given finiterank perturbation of the identity.

\section{COROLLARY 4.14}

Let $X$ be a separable reflexive Banach space, and let $G \leq \mathrm{GL}(X)$ be a bounded subgroup containing a nontrivial finite-rank perturbation of the identity.

- If there are no nonzero totally bounded $G$-orbits, then $X$ admits a complemented $G$-invariant subspace with a Schauder basis.

- If $X$ admits no nontrivial $G$-invariant decompositions, then $X$ itself has a Schauder basis.

The first known example of a (separable) space such that no complemented subspace has a basis or even a finite-dimensional decomposition was given in 1983 by Pisier's famous counterexample to a conjecture of Grothendieck (see [53]). More recently, Allexandrov, Kutzarova, and Plichko [2] observed that the Gowers-Maurey constructions of hereditarily indecomposable spaces (see [35]) implied the existence of separable reflexive spaces without any Schauder decomposition.

COROLLARY 4.15

Let $X$ be a separable reflexive Banach space, and let $G \leq \mathrm{GL}_{f}(X)$ be a bounded subgroup, strongly closed in $\mathrm{GL}(X)$. Then either $G$ acts nearly trivially on $X$ or $X$ has a complemented subspace with a Schauder basis. 
Proof

By Theorem 4.10, $X=K_{G} \oplus\left(K_{G^{*}}\right)_{\perp}$ and $\left(K_{G^{*}}\right)_{\perp}$ is either infinite-dimensional or $X=K_{G}$. If $X=K_{G}$, then $G$ is almost periodic and hence acts nearly trivially on $X$ by Theorem 3.11.

On the other hand, if $\left(K_{G^{*}}\right)_{\perp}$ is infinite-dimensional, then

$$
\left.G\right|_{\left(K_{G^{*}}\right)_{\perp}}=\left\{\left.T\right|_{\left(K_{G^{*}}\right)_{\perp}} \mid T \in G\right\}
$$

is a bounded subgroup of $\mathrm{GL}_{f}\left(\left(K_{G^{*}}\right)_{\perp}\right)$ having no nonzero relatively compact orbits. By Theorem 4.13, $\left(K_{G^{*}}\right)_{\perp}$ and thus also $X$ has a complemented subspace with a Schauder basis.

\section{THEOREM 4.16}

Let $X$ be a separable reflexive Banach space, let $G \leq \mathrm{GL}_{f}(X)$ be a bounded subgroup, and let $X=H_{G} \oplus\left(H_{G^{*}}\right)_{\perp}$ be the corresponding Alaoglu-Birkhoff decomposition. Then $\left(H_{G^{*}}\right)_{\perp}$ is finite-dimensional or admits a finite-dimensional decomposition.

Proof

Renorm so that $G$ is a group of isometries, and let $T_{1}, T_{2}, \ldots$ list the elements of a dense subgroup of $G$. Then $\left(H_{G^{*}}\right)_{\perp}=\overline{\operatorname{span}}\left(\bigcup_{n \geq 1} F_{T_{n}}\right)$ is complemented in $X$ and admits a finite-dimensional decomposition.

\section{THEOREM 4.17}

Let $X$ be a separable reflexive Banach space, let $G \leq \mathrm{GL}_{f}(X)$ be a bounded subgroup, and let $X=H_{G} \oplus\left(H_{G^{*}}\right)_{\perp}$ be the corresponding Alaoglu-Birkhoff decomposition. Then $\left(H_{G^{*}}\right)_{\perp}$ admits a $G$-invariant Schauder decomposition

$$
\left(H_{G^{*}}\right)_{\perp}=Y_{1} \oplus Y_{2} \oplus \cdots
$$

(possibly with finitely many summands) such that each $Y_{n}$ has a (possibly finite) Schauder basis.

Proof

By renorming, we can suppose that $G$ is a group of isometries of $X$. Let also $T_{1}, T_{2}$, ... list a dense subset of $G$.

We now apply Theorem 4.13 (and the comments following it) first to the $G$-invariant subspace $\left(H_{G^{*}}\right)_{\perp}$ and the isometry $T_{1}$ to obtain a $G$-invariant decomposition,

$$
\left(H_{G^{*}}\right)_{\perp}=Y_{1} \oplus Z_{1},
$$


where the projection onto $Z_{1}$ has norm $1, F_{T_{1}} \subseteq Y_{1}$, and $Y_{1}$ has a (possibly finite) Schauder basis.

Let now $G_{1}=\left\{\left.T\right|_{Z_{1}} \mid T \in G\right\}$ denote the restriction of $G$ to $Z_{1}$, and note that $G_{1}$ has no nonzero fixed points on $Z_{1}$. We can therefore apply Theorem 4.13 to $G_{1} \leq \mathrm{GL}_{f}\left(Z_{1}\right), Z_{1}$ and the first nontrivial isometry $T$ in the list $T_{2}\left|Z_{1}, T_{3}\right| Z_{1}, \ldots$ to obtain a $G_{1}$-invariant, and thus also $G$-invariant, decomposition

$$
Z_{1}=Y_{2} \oplus Z_{2}
$$

where the projection of $Z_{1}$ onto $Z_{2}$ has norm $1, F_{T} \subseteq Y_{2}$, and $Y_{2}$ has a (possibly finite) Schauder basis.

Continuing in this manner, we construct a (possibly finite) list of $G$-invariant closed subspaces $Y_{1}, Y_{2}, \ldots$ and $Z_{1}, Z_{2}, \ldots$ such that $\left(H_{G^{*}}\right)_{\perp}=Y_{1} \oplus Z_{1}$, $Z_{n}=Y_{n+1} \oplus Z_{n+1}$ and the projection of $\left(H_{G^{*}}\right)_{\perp}$ onto $Z_{n}$ has norm 1 for all $n$. Moreover, by construction, we have $F_{T_{n}} \subseteq Y_{1} \oplus \cdots \oplus Y_{n}$, whereby $\left(H_{G^{*}}\right)_{\perp}=$ $\overline{\operatorname{span}}\left(\bigcup_{n \geq 1} F_{T_{n}}\right) \subseteq Y_{1} \oplus Y_{2} \oplus \cdots$. It follows that $\left(H_{G^{*}}\right)_{\perp}$ admits the $G$-invariant Schauder decomposition

$$
\left(H_{G^{*}}\right)_{\perp}=Y_{1} \oplus Y_{2} \oplus \cdots,
$$

where the norms of the projections onto the initial segments are bounded by 2 .

\section{Groups of finite-dimensional isometries}

\subsection{Norm-closed subgroups of $\mathrm{GL}_{f}(X)$}

By Theorem 4.10, the study of the isometry group of a separable reflexive Banach space essentially reduces to the study of two separate cases, namely, when all orbits are totally bounded and when no orbit is totally bounded. We now treat the second case.

LEMMA 5.1

Suppose that $G$ is a group acting by isometries on a complete metric space $M$. Then the following conditions are equivalent:

(1) No orbit is totally bounded.

(2) For every compact $C \subseteq M$, there is a $g \in G$ such that $g[C] \cap C=\emptyset$.

(3) For any compact $C \subseteq M$ and $g \in G$ there are $f_{1}, f_{2} \in G$ such that $g=f_{1} f_{2}$ and $f_{i}[C] \cap C=\emptyset$.

\section{Proof}

That (3) implies (1) is trivial. For the implication from (1) to (2), we will use the following well-known fact from group theory. 
CLAIM 5.2

Suppose that $F_{1} E_{1} \cup \cdots \cup F_{n} E_{n}$ is a covering of a group $G$, where $E_{i}, F_{i}$ are subsets of $G$ with $F_{i}$ finite. Then there is a finite set $F \subseteq G$ and an $i$ such that $G=F E_{i} E_{i}^{-1}$.

\section{Proof}

The proof is by induction on $n$, the case $n=1$ being trivial. So suppose that the result holds for $n-1$, and let $G=F_{1} E_{1} \cup \cdots \cup F_{n} E_{n}$ be a covering. Then, if $G \neq$ $F_{1} E_{1} E_{1}^{-1}$, pick $g \in G \backslash F_{1} E_{1} E_{1}^{-1}$, whereby $g E_{1} \cap F_{1} E_{1}=\emptyset$. It follows that $g E_{1} \subseteq$ $F_{2} E_{2} \cup \cdots \cup F_{n} E_{n}$ and hence that

$$
F_{1} E_{1}=F_{1} g^{-1} \cdot g E_{1} \subseteq F_{1} g^{-1} F_{2} E_{2} \cup \cdots \cup F_{1} g^{-1} F_{n} E_{n} .
$$

Thus,

$$
G=\left(F_{1} g^{-1} F_{2} \cup F_{2}\right) E_{2} \cup \cdots \cup\left(F_{1} g^{-1} F_{n} \cup F_{n}\right) E_{n},
$$

finishing the inductive step.

Assume now that (1) holds, and, for any $x \in M$ and $\delta>0$, define $V(x, \delta)=\{g \in$ $G \mid d(g x, x)<\delta\}$ and note that for any $f \in G$,

$$
\begin{aligned}
f \cdot V(x, \delta) \cdot V(x, \delta)^{-1} & =f \cdot V(x, \delta) \cdot V(x, \delta) \subseteq f \cdot V(x, 2 \delta) \\
& =\{g \in G \mid d(g x, f x)<2 \delta\} .
\end{aligned}
$$

Suppose that $C \subseteq M$ is compact, and assume toward a contradiction that $g[C] \cap C \neq$ $\emptyset$ for any $g \in G$. Since no orbit is totally bounded, pick $\delta>0$ such that the orbit of no point of $C$ admits a finite covering by sets of diameter $8 \delta$, and let

$$
B\left(x_{1}, \delta\right) \cup \cdots \cup B\left(x_{n}, \delta\right)
$$

be a finite subcover of the cover $\bigcup_{x \in C} B(x, \delta)$ of $C$. Then for any $g$ there are $i, j$ such that $g\left[B\left(x_{i}, \delta\right)\right] \cap B\left(x_{j}, \delta\right) \neq \emptyset$, whence $d\left(g x_{i}, x_{j}\right)<2 \delta$. Now, for every $i, j$ pick if possible some $f \in G$ such that $d\left(f x_{i}, x_{j}\right)<2 \delta$, and let $F \subseteq G$ be the finite set of such $f$. Then

$$
G=F \cdot V\left(x_{1}, 4 \delta\right) \cup \cdots \cup F \cdot V\left(x_{n}, 4 \delta\right),
$$

and so by Claim 5.2 there is some finite $E \subseteq G$ and $i$ such that $G=E \cdot V\left(x_{i}, 8 \delta\right)$, whence $G \cdot x_{i}$ is covered by finitely many open balls of diameter $8 \delta$, contradicting the choice of $\delta$.

Now, to see that (2) implies (3), suppose that $g \in G$ and $C \subseteq M$ is compact. Then there is some $f_{1} \in G$ such that

$$
f_{1}[C \cup g[C]] \cap(C \cup g[C])=\varnothing,
$$


whence, in particular, $f_{1}[C] \cap C=\emptyset$. Letting $f_{2}=f_{1}^{-1} g$, we have

$$
f_{2}[C] \cap C=f_{1}^{-1} g[C] \cap C \subseteq f_{1}^{-1}[C \cup g[C]] \cap(C \cup g[C])=\emptyset .
$$

Since $g=f_{1} f_{2}$, this finishes the proof.

The following lemma is an immediate corollary.

\section{LEMMA 5.3}

Let $X$ be a Banach space, and let $G$ be a group of isometries of $X$ such that no nonzero $G$-orbit is totally bounded. Then for any finite-dimensional subspace $F$ of $X$ there exists a $T$ in $G$ such that $F \cap T[F]=\{0\}$. In fact, for any finite-dimensional subspace $F$ of $X$, any isometry $S$ in $G$ can be written as a product of two such $T$ in $G$.

The goal is now to provide a structure result for norm-closed groups of finitedimensional isometries for which we will need a couple of lemmas.

LEMMA 5.4

Let $X$ be a Banach space, and let $T, U \in \mathscr{L}(X)$. Then there exists an injective map from $H_{T U} /\left(H_{T} \cap H_{U}\right)$ into $F_{T} \cap F_{U}$. In particular, if $F_{T} \cap F_{U}=\{0\}$, then $H_{T U}=$ $H_{T} \cap H_{U}$.

\section{Proof}

Obviously $H_{T} \cap H_{U} \subseteq H_{T U}$, and $U-$ Id induces a map from $H_{T U} /\left(H_{T} \cap H_{U}\right)$ into $F_{U}$. For any $x \in H_{T U}$,

$$
(U-\mathrm{Id}) x=(U-T U) x=(\mathrm{Id}-T)(U x) \in F_{T},
$$

so the map induced by $U$ - Id takes its values in $F_{T} \cap F_{U}$.

Finally, if $(U-\mathrm{Id}) x=0$ for $x \in H_{T U}$, then $U x=x$ and $T x=T U x=x$, so $x \in H_{T} \cap H_{U}$. This proves that the map induced by $U-\mathrm{Id}$ is injective.

\section{LEMMA 5.5}

Let $X$ be a Banach space, and let $T, U \in \mathrm{GL}_{f}(X)$. Then

$$
\left|\operatorname{dim} F_{T}-\operatorname{dim} F_{U}\right| \leq \operatorname{dim} F_{T U} \leq \operatorname{dim} F_{T}+\operatorname{dim} F_{U} .
$$

\section{Proof}

Since $T U-\mathrm{Id}=(T-\mathrm{Id})+T(U-\mathrm{Id})$, we see that $F_{T U} \subseteq F_{T}+T\left[F_{U}\right]$ and therefore that

$$
\operatorname{dim} F_{T U} \leq \operatorname{dim} F_{T}+\operatorname{dim} T\left[F_{U}\right]=\operatorname{dim} F_{T}+\operatorname{dim} F_{U}
$$


Applying this to $T U$ and $U^{-1}$, it follows that

$$
\operatorname{dim} F_{T} \leq \operatorname{dim} F_{T U}+\operatorname{dim} F_{U^{-1}}=\operatorname{dim} F_{T U}+\operatorname{dim} F_{U},
$$

and so $\operatorname{dim} F_{T U} \geq \operatorname{dim} F_{T}-\operatorname{dim} F_{U}$. Since also

$$
\operatorname{dim} F_{T U}=\operatorname{dim} F_{U^{-1} T^{-1}} \geq \operatorname{dim} F_{U^{-1}}-\operatorname{dim} F_{T^{-1}}=\operatorname{dim} F_{U}-\operatorname{dim} F_{T},
$$

we find that $\operatorname{dim} F_{T U} \geq\left|\operatorname{dim} F_{T}-\operatorname{dim} F_{U}\right|$.

LEMMA 5.6

Let $X$ be a Banach space, and let $G \leq \mathrm{GL}_{f}(X)$ be a bounded subgroup, norm closed in $\mathrm{GL}(X)$. Then for any nonempty, norm-open $U \subseteq G$, there is a smaller norm-open set $\emptyset \neq \mathcal{V} \subseteq U$ such that $\operatorname{dim} F_{T}$ is constant for $T \in \mathcal{V}$.

Proof

We work in the norm topology. Note that for any $n$ the set

$$
D_{n}=\{A \in \mathscr{L}(X) \mid \operatorname{dim}(\operatorname{im} A) \leq n\}
$$

is closed, whence

$$
E_{n}=\left\{T \in G \mid \operatorname{dim} F_{T} \leq n\right\}=G \cap\left(\mathrm{Id}+D_{n}\right)
$$

is closed in $G$. Now, if $U \subseteq G$ is nonempty open, then as $G=\bigcup_{n \geq 1} E_{n}$, it follows from the Baire category theorem in $\mathcal{U}$ that some $E_{n} \cap \mathcal{U}$ has nonempty interior. Moreover, if $n$ is minimal with this property, we see that $\mathcal{V}=\operatorname{int}\left(E_{n} \cap \mathcal{U}\right) \backslash E_{n-1} \neq \varnothing$ and $\operatorname{dim} F_{T}=n$ for all $T \in \mathcal{V}$.

From this we deduce the following lemma.

\section{LEMMA 5.7}

Let $X$ be a Banach space, and let $G \leq \mathrm{GL}_{f}(X)$ be a bounded subgroup, norm closed in $\operatorname{GL}(X)$. Then there are $\delta>0$ and a constant $N$ such that for all $T, U \in G$,

$$
\|T-U\|<\delta \Rightarrow\left|\operatorname{dim} F_{T}-\operatorname{dim} F_{U}\right| \leq N .
$$

It follows that if $G$ is norm compact, then

$$
\sup \left\{\operatorname{dim} F_{T} \mid T \in G\right\}<\infty .
$$

\section{Proof}

Without loss of generality, $G \leq \operatorname{Isom}_{f}(X)$. By Lemma 5.6, there are $S \in G$ and $\delta>0$ such that $\operatorname{dim} F_{T}=\operatorname{dim} F_{S}$ whenever $\|T-S\| \leq \delta$. It follows that if $T, U \in G$ satisfy 
$\|T-U\|<\delta$ and thus $\left\|S T U^{-1}-S\right\|<\delta$, then $\operatorname{dim} F_{S T U^{-1}}=\operatorname{dim} F_{S}$ and so, by Lemma 5.5,

$$
\begin{aligned}
\left|\operatorname{dim} F_{T}-\operatorname{dim} F_{U}\right| & =\left|\operatorname{dim} F_{T}-\operatorname{dim} F_{U^{-1}}\right| \\
& \leq \operatorname{dim} F_{T U^{-1}} \\
& \leq \operatorname{dim} F_{S^{-1}}+\operatorname{dim} F_{S T U^{-1}} \\
& =2 \operatorname{dim} F_{S} .
\end{aligned}
$$

Setting $N=2 \operatorname{dim} F_{S}$, the result follows.

If now $G$ is norm compact, it can be covered by a finite number of open balls of radius $\delta$, whereby $\operatorname{dim} F_{T}$ is bounded above for $T \in G$.

LEMMA 5.8

Let $X$ be a Banach space with a Gâteaux differentiable norm, and let $T$ be a finitedimensional isometry on $X$. Then

$$
F_{T}=J\left[H_{T}\right]_{\perp} .
$$

Proof

From Lemma 4.3 we easily deduce that $J\left[H_{T}\right] \subseteq H_{T^{*}}$ and therefore, as $F_{T}$ is closed,

$$
F_{T}=\left(F_{T}^{\perp}\right)_{\perp}=\left(H_{T^{*}}\right)_{\perp} \subseteq J\left[H_{T}\right]_{\perp} .
$$

It follows by Lemma 4.1(a) that

$$
X=H_{T} \oplus F_{T} \subseteq H_{T} \oplus J\left[H_{T}\right]_{\perp} \subseteq X,
$$

whence $F_{T}=J\left[H_{T}\right]_{\perp}$.

\section{THEOREM 5.9}

Let $X$ be a Banach space with a Gâteaux differentiable norm, let $G \leq \operatorname{Isom}_{f}(X)$ be norm closed in $\mathrm{GL}(X)$, and assume that no nonzero point of $X$ has a totally bounded $G$-orbit. Then $G$ is discrete in the norm topology and hence is locally finite; that is, any finitely generated subgroup is finite.

Proof

We work in the norm topology on $G$. Assume toward a contradiction that $G$ is not discrete. Then for every neighborhood $W$ of Id there is a finite-dimensional isometry $U \in \mathcal{W}$ with $\operatorname{dim} F_{U} \geq 1$. By Lemma 5.7, let $n \geq 1$ be minimal such that for some neighborhood $\mathcal{V}$ of Id in $G$ we have $\operatorname{dim}\left(F_{U}\right) \leq n$ for all $U \in \mathcal{V}$. Let $\mathcal{U}$ be a conjugacy-invariant neighborhood of Id in $G$ such that $U^{2} \subseteq \mathcal{V}$, and choose an isometry $T \in U$ such that $\operatorname{dim}\left(F_{T}\right)=n$. 
Suppose first that $S$ is an isometry such that $S\left[F_{T}\right] \cap F_{T}=\{0\}$. Then, since $F_{S T S^{-1}}=S\left[F_{T}\right]$, Lemma 5.4 implies that $H_{T S T S^{-1}}=H_{T} \cap H_{S T S^{-1}}$. However, $T \in \mathcal{U}$ and $\mathcal{U}$ is conjugacy invariant, so $T S T S^{-1} \in \mathcal{U}^{2} \subseteq \mathcal{V}$, and thus

$$
\begin{aligned}
\operatorname{codim}\left(H_{T}\right) & =\operatorname{dim}\left(F_{T}\right) \\
& =n \\
& \geq \operatorname{dim}\left(F_{T S T S^{-1}}\right) \\
& =\operatorname{codim}\left(H_{T S T S^{-1}}\right) \\
& =\operatorname{codim}\left(H_{T} \cap H_{S T S^{-1}}\right),
\end{aligned}
$$

whence, as $\operatorname{codim}\left(H_{T}\right)=\operatorname{codim}\left(H_{S T S^{-1}}\right)$, we have $H_{T}=H_{S T S^{-1}}=S\left[H_{T}\right]$.

Now, by Lemma 5.3, any isometry $U$ can be written as a product of two isometries $S_{1}, S_{2}$ in $G$ such that $S_{i}\left[F_{T}\right] \cap F_{T}=\{0\}$. By the above calculation, $H_{T}$ is $S_{i}$-invariant and therefore also $U$-invariant. On the other hand, by Lemma 4.3, we have that $U^{*} J\left[H_{T}\right]=J U^{-1}\left[H_{T}\right]=J\left[H_{T}\right]$, so, as $U$ is arbitrary, $J\left[H_{T}\right]$ is $G$-invariant.

Since, by Lemma $5.8, F_{T}=J\left[H_{T}\right]_{\perp}$, it is a nontrivial, finite-dimensional, $G$-invariant subspace of $X$, contradicting that no orbit is totally bounded.

To see that $G$ is locally finite, note that by Lemma 3.8 any finitely generated sub-

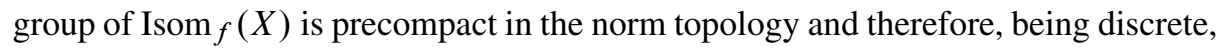
must be finite.

THEOREM 5.10

Let $X$ be a Banach space with separable dual, let $G \leq \mathrm{GL}_{f}(X)$ be a bounded subgroup, norm closed in $\mathrm{GL}(X)$, and assume that no nonzero point of $X$ has a totally bounded $G$-orbit. Then $G$ is discrete and locally finite in the norm topology.

\section{Proof}

By renorming $X$, we may assume that $G \leq \operatorname{Isom}_{f}(X)$. Thus, by Proposition 4.6 and the fact that a norm whose dual norm is LUR is Gâteaux differentiable, we can also suppose that the norm on $X$ is Gâteaux differentiable, so the result follows from Theorem 5.9.

\subsection{Decompositions by strongly closed subgroups of $\mathrm{GL}_{f}(X)$}

\section{THEOREM 5.11}

Suppose that $X$ is a separable reflexive Banach space and that $G \leq \mathrm{GL}_{f}(X)$ is bounded and strongly closed in $\mathrm{GL}(X)$. Let also $G_{0} \leq G$ denote the connected component of the identity in $G$. 
Then $G_{0}$ acts nearly trivially on $X$ and therefore is a compact Lie group. Moreover, $G_{0}$ is open in $G$, while $G / G_{0}$ is a countable, locally finite and thus amenable group. It follows that $G$ is an amenable Lie group.

Furthermore, $X$ admits a $G$-invariant decomposition $X=X_{1} \oplus X_{2} \oplus X_{3} \oplus X_{4}$, where

(1) no nonzero point of $X_{1}$ has a relatively compact $G$-orbit;

(2) every $G$-orbit on $X_{2} \oplus X_{3} \oplus X_{4}$ is relatively compact;

(3) $X_{4}$ is the subspace of points which are fixed by $G$;

(4) $X_{3}$ is finite-dimensional or has a finite-dimensional decomposition;

(5) $\quad X_{2}$ is finite-dimensional and $X_{1} \oplus X_{3} \oplus X_{4}$ is the subspace of points which are fixed by $G_{0}$;

(6) if $X_{1}=\{0\}$, then $G$ acts nearly trivially on $X$;

(7) if $X_{1} \neq\{0\}$, then $X_{1}$ is infinite-dimensional, has a finite-dimensional decomposition, and admits a $G$-invariant Schauder decomposition (possibly with finitely many terms)

$$
X_{1}=Y_{1} \oplus Y_{2} \oplus \cdots,
$$

where every $Y_{i}$ has a Schauder basis.

The norm of the associated projection onto $X_{4}$ (resp., $X_{1}, X_{2}, X_{3}$ ) is at most $\|G\|^{2}$ (resp., $2\|G\|^{2}$ ), and in (7) the finite-dimensional decomposition and the $G$-invariant Schauder decomposition both have constant at most $2\|G\|^{2}$, where $\|G\|=\sup _{g \in G}\|g\|$.

\section{Proof}

Note that, by Proposition 3.5, since $X^{*}$ is separable and $G \leq \mathrm{GL}_{f}(X)$, the norm and strong operator topologies coincide on $G$.

Let now $X=K_{G} \oplus\left(K_{G^{*}}\right)_{\perp}$ be the $G$-invariant decomposition given by Theorem 4.10, and, for simplicity of notation, let $X_{1}=\left(K_{G^{*}}\right)_{\perp}$.

We claim that $\left.G\right|_{X_{1}}=\left\{\left.T\right|_{X_{1}} \mid T \in G\right\}$ is a strongly closed subgroup of $\operatorname{GL}\left(X_{1}\right)$. To see this, suppose that $T_{n} \in G$ and $T \in \mathrm{GL}\left(X_{1}\right)$ are such that $\left.T_{n}\right|_{X_{1}} \underset{\text { soT }}{\longrightarrow} T$. By Proposition 3.2, $\left.G\right|_{K_{G}}=\left\{\left.T\right|_{K_{G}} \mid T \in G\right\} \leq \mathrm{GL}\left(K_{G}\right)$ is precompact in the strong operator topology and thus $\left(\left.T_{n}\right|_{K_{G}}\right)_{n \in \mathbb{N}}$ has a subsequence $\left(\left.T_{n_{i}}\right|_{K_{G}}\right)_{i \in \mathbb{N}}$ converging in the strong operator topology to an operator $S \in \mathrm{GL}\left(K_{G}\right)$, whence $T_{n_{i}} \underset{\text { SOT }}{\longrightarrow} S \oplus$ $T \in \mathrm{GL}\left(K_{G} \oplus X_{1}\right)$. Since $G$ is closed in the strong operator topology, we see that $S \oplus T \in G$, whence $T=\left.\left.(S \oplus T)\right|_{X_{1}} \in G\right|_{X_{1}}$, showing that $\left.G\right|_{X_{1}}$ is strongly closed.

Now, by Proposition 3.5, since $X_{1}^{*}$ is separable and $\left.G\right|_{X_{1}} \leq \mathrm{GL}_{f}\left(X_{1}\right)$, the norm and strong operator topologies coincide on $\left.G\right|_{X_{1}}$. Moreover, as there is no nonzero relatively compact $\left.G\right|_{X_{1}}$-orbit on $X_{1}$, Theorem 5.10 implies that $\left.G\right|_{X_{1}}$ is discrete and locally finite. Being separable, it must be countable. Moreover, since the map 
$\left.\left.T \in G \mapsto T\right|_{X_{1}} \in G\right|_{X_{1}}$ is strongly continuous, its kernel $G^{\prime}=\left\{T \in G \mid X_{1} \subseteq H_{T}\right\}$ is a countable index, closed normal subgroup of the Polish group $G$ and thus must be open by the Baire category theorem. Since every $G^{\prime}$-orbit on $X$ is relatively compact, Proposition 3.2 implies that $G^{\prime}={\overline{G^{\prime}}}^{\text {SOT }}$ is compact in the strong operator topology and so, by Theorem 3.11, $G^{\prime}$ acts nearly trivially on $X$, and since $G_{0} \leq G^{\prime}$, the same holds for $G_{0}$. It follows that $G^{\prime}$ is a compact Lie group, whence also that $G$ is Lie, and that $G_{0}$ is a compact, open, normal subgroup in $G$. Since $G^{\prime}$ is compact, $\left[G^{\prime}: G_{0}\right]<\infty$, and so $G / G_{0}$ is an extension of the finite group $G^{\prime} / G_{0}$ by the locally finite group $G / G^{\prime}$ and thus is itself locally finite. Thus, as both $G_{0}$ and $G / G_{0}$ are amenable, so is $G$.

We now consider the canonical complement $K_{G}=H_{G} \oplus\left(\left(H_{G^{*}}\right)_{\perp} \cap K_{G}\right)$ to $X_{1}=\left(K_{G^{*}}\right)_{\perp}$. Set $Y=\left(\left(H_{G^{*}}\right)_{\perp} \cap K_{G}\right)$, and let $\pi: G \rightarrow \operatorname{GL}(Y)$ be the strongly continuous representation $\pi(T)=\left.T\right|_{Y}$. Since $\left.T\right|_{X_{1}}=\operatorname{Id}_{X_{1}}$ for all $T \in G_{0}, \pi\left(G_{0}\right)$ is strongly closed in $\operatorname{GL}(Y)$, and so, by Theorem 3.11, $Y$ admits a $G_{0}$-invariant decomposition

$$
Y=H_{\pi\left(G_{0}\right)} \oplus\left(H_{\pi\left(G_{0}\right)^{*}}\right)_{\perp},
$$

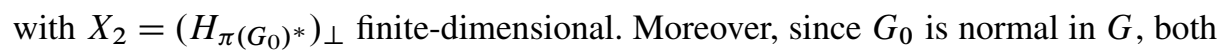

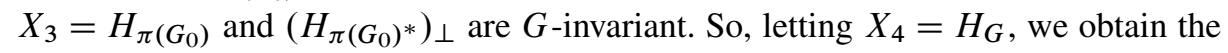
desired decomposition.

If $\left(K_{G^{*}}\right)_{\perp}=X_{1} \neq\{0\}$, then $X_{1}$ is infinite-dimensional and must have a finitedimensional decomposition by Theorem 4.16. Similarly, the existence of the $G$-invariant decomposition into subspaces with Schauder bases follows from Theorem 4.17 plus the fact that there are no nontrivial $G$-invariant finite-dimensional subspaces of $X_{1}$. On the other hand, if $X_{1}=\{0\}$, then $G$ is almost periodic and therefore acts nearly trivially on $X$ by Theorem 3.11 .

\section{Spaces with a small algebra of operators}

\subsection{Unconditional sequences of eigenvectors}

LEMMA 6.1

Let $0<\theta<\epsilon<\pi$ and $0 \leq \alpha \leq 2 \pi$. For any interval $I \subseteq \mathbb{N}$ of cardinality at least $3 \pi / \theta+1$, there exists a subinterval $J \subseteq I$ of cardinality at least $\epsilon / \theta$ such that for all $m \in J, d(m \theta, \alpha+2 \pi \mathbb{N})<\epsilon$.

\section{Proof}

Let $\left(u_{n}\right)_{n \in I}$ be the finite sequence $(n \theta-\alpha)_{n \in I}$, and note that the difference between the first and last element of $u_{n}$ is at least $3 \pi$, while the difference between two successive elements is at most $\theta<\pi$. Write the first element of the sequence as $k 2 \pi+\alpha_{0}$, 
for some $\alpha_{0} \in\left[0,2 \pi\right.$ [, and let $\alpha^{\prime}=k 2 \pi+\alpha$ if $\alpha_{0} \leq \alpha$, or $\alpha^{\prime}=(k+1) 2 \pi+\alpha$ otherwise.

Let $n_{1}$ be the largest element of $I$ such that $u_{n_{1}} \leq \alpha^{\prime}$, and note that the difference between $u_{n_{1}}$ and the first element of the sequence is at most $2 \pi$. Now consider $p \in \mathbb{N}$ maximal with $1 \leq p<\epsilon / \theta$, and note that $u_{n_{1}+p}-u_{n_{1}} \leq p \theta<\epsilon$. Therefore, since $\alpha^{\prime} \in\left[u_{n_{1}}, u_{n_{1}+p}\right], d\left(u_{n}, \alpha^{\prime}\right)=d(n \theta, \alpha+2 \pi \mathbb{N})<\epsilon$ for any $n$ in the interval $J=$ $\left\{n_{1}, \ldots, n_{1}+p\right\}$. This interval has cardinality at least $\epsilon / \theta$. The difference between the first $u_{n}$ and $u_{n_{1}+p}$ is at most $2 \pi+p \theta \leq 2 \pi+\epsilon<3 \pi$; therefore, $J$ is a subinterval of $I$.

LEMMA 6.2

Let $\left(\epsilon_{k}\right)_{k \geq 1}$ be a sequence of real numbers in the interval $] 0, \pi\left[\right.$. Let $\left(\theta_{k}\right)_{k \geq 1}$ be a sequence of positive real numbers such that $\epsilon_{1} / \theta_{1} \geq 1$ and $\epsilon_{k} / \theta_{k}>3 \pi / \theta_{k-1}+1$ for all $k>1$. Let $n \in \mathbb{N}$, and let $\left(\alpha_{k}\right)_{1 \leq k \leq n}$ be a finite sequence of real numbers. Then there exists some $m \in \mathbb{N}$ such that for all $k=1, \ldots, n$,

$$
d\left(m \theta_{k}, \alpha_{k}+2 \pi \mathbb{N}\right)<\epsilon_{k} .
$$

\section{Proof}

First, we may by the previous lemma pick an interval $J_{n}$ of cardinality at least $\epsilon_{n} / \theta_{n}$ such that for all $m \in J_{n}, d\left(m \theta_{n}-\alpha_{n}, 2 \pi \mathbb{N}\right)<\epsilon_{n}$. Since $\epsilon_{n} / \theta_{n}-1>3 \pi / \theta_{n-1}$, the lemma applies again to obtain an interval $J_{n-1} \subseteq J_{n}$ of cardinality at least $\epsilon_{n-1} / \theta_{n-1}$ such that for all $m \in J_{n-1}, d\left(m \theta_{n-1}-\alpha_{n-1}, 2 \pi \mathbb{N}\right)<\epsilon_{n-1}$. After $n$ steps we have obtained the desired result for any $m$ in some interval $J_{1}$ of cardinality at least $\epsilon_{1} / \theta_{1}$ and therefore nonempty by hypothesis.

\section{PROPOSITION 6.3}

Let $T$ be a linear contraction on a complex Banach space $X$ having infinitely many eigenvalues of modulus 1 . Then, for any $\epsilon>0, X$ contains a $(1+\epsilon)$-unconditional basic sequence.

\section{Proof}

Modulo replacing $T$ by some $\lambda T,|\lambda|=1$, we can suppose that there are distinct $\theta_{n}$ converging to zero such that each $e^{i \theta_{n}}$ is an eigenvalue for $T$ with corresponding normalized eigenvector $x_{n} \in X$. Moreover, without loss of generality, we can also assume that $0<\theta_{n}<\pi$ and that, given $\epsilon_{n}>0$ such that $\sum_{n=1}^{\infty} \epsilon_{n} \leq \delta$, where $\frac{2+\delta}{2-\delta}<$ $1+\epsilon$, one has $\epsilon_{1} / \theta_{1} \geq 1$ and $\epsilon_{n} / \theta_{n}>3 \pi / \theta_{n-1}+1$ for all $n>1$.

Suppose that $N \geq 1$, and consider a vector of the form $x=\sum_{n \leq N} \lambda_{n} x_{n}$ for $\lambda_{n} \in \mathbb{C}$. Now, if $e^{i \alpha_{n}} \in \mathbb{T}$ and $m \geq 1$, we have 


$$
T^{m}(x)=\sum_{n \leq N} e^{i m \theta_{n}} \lambda_{n} x_{n},
$$

and so

$$
\left\|T^{m}(x)-\sum_{n \leq N} e^{i \alpha_{n}} \lambda_{n} x_{n}\right\|=\left\|\sum_{n \leq N}\left(e^{i m \theta_{n}}-e^{i \alpha_{n}}\right) \lambda_{n} x_{n}\right\| \leq \sum_{n \leq N}\left|1-e^{i\left(\alpha_{n}-m \theta_{n}\right)}\right|\left|\lambda_{n}\right| .
$$

Choosing $m$ according to Lemma 6.2 so that $d\left(m \theta_{n}-\alpha_{n}, 2 \pi \mathbb{N}\right)<\epsilon_{n}$ for each $n \leq N$, we conclude that

$$
\left\|T^{m}(x)-\sum_{n \leq N} e^{i \alpha_{n}} \lambda_{n} x_{n}\right\| \leq \sum_{n \leq N} \epsilon_{n}\left|\lambda_{n}\right| \leq \delta \cdot \sup _{n \leq N}\left|\lambda_{n}\right|
$$

and so also that

$$
\left\|\sum_{n \leq N} e^{i \alpha_{n}} \lambda_{n} x_{n}\right\| \leq\left\|\sum_{n \leq N} \lambda_{n} x_{n}\right\|+\delta \cdot \sup _{n \leq N}\left|\lambda_{n}\right|
$$

Let $n_{0} \leq N$ be chosen such that $\left|\lambda_{n_{0}}\right|=\sup _{n \leq N}\left|\lambda_{n}\right|$, and set $e^{i \alpha_{n_{0}}}=1$ and $e^{i \alpha_{n}}=$ -1 for $n \neq n_{0}$. By (2), we then have

$$
\begin{aligned}
2\left|\lambda_{n_{0}}\right| & \leq\left\|\lambda_{n_{0}} x_{n_{0}}+\sum_{\substack{n \leq N \\
n \neq n_{0}}} \lambda_{n} x_{n}\right\|+\left\|\lambda_{n_{0}} x_{n_{0}}-\sum_{\substack{n \leq N \\
n \neq n_{0}}} \lambda_{n} x_{n}\right\| \\
& \leq 2\left\|\sum_{n \leq N} \lambda_{n} x_{n}\right\|+\delta \cdot\left|\lambda_{n_{0}}\right|,
\end{aligned}
$$

that is, $\sup _{n \leq N}\left|\lambda_{n}\right|=\left|\lambda_{n_{0}}\right| \leq \frac{2}{2-\delta}\left\|\sum_{n \leq N} \lambda_{n} x_{n}\right\|$. Combining this with (2), for any $\lambda_{n}$ and $e^{i \alpha_{n}} \in \mathbb{T}$, we have

$$
\left\|\sum_{n \leq N} e^{i \alpha_{n}} \lambda_{n} x_{n}\right\| \leq \frac{2+\delta}{2-\delta}\left\|\sum_{n \leq N} \lambda_{n} x_{n}\right\|
$$

which shows that $\left(x_{n}\right)_{n=1}^{\infty}$ is a $(1+\epsilon)$-unconditional basic sequence.

We refer the reader to [50] for results about the existence of 1-unconditional basic sequences in a similar setting.

With this in hand, we may prove the following.

THEOREM 6.4

Let $X$ be a Banach space containing no unconditional basic sequence. Then any bounded subgroup $G \leq \mathrm{GL}_{i n}(X)$ is contained in $\mathrm{GL}_{f}(X)$ and so, in particular,

$$
\operatorname{Isom}_{a f}(X)=\operatorname{Isom}_{f}(X) .
$$




\section{Proof}

By Theorem 3.4, it suffices to prove that any bounded subgroup $G \leq \mathrm{GL}_{a f}(X)$ is contained in $\mathrm{GL}_{f}(X)$ and, by renorming, that any almost finite-dimensional isometry is finite-dimensional.

Assume first that $X$ is complex. Since almost finite-rank operators are Riesz operators, the spectrum of an almost finite-dimensional isometry $T$ of $X$ is either a finite or an infinite sequence of distinct eigenvalues with finite multiplicity, together with the value 1 , which is the limit of the sequence in the infinite case (see [35]). Since $X$ does not contain an unconditional basic sequence, by Proposition 6.3, the second case cannot occur and so $\sigma(T)$ is finite. Lemma 3.12 then implies that $T$ - Id must have finite rank.

If instead $X$ is real, we claim that the complexification $\hat{X}$ of $X$ does not contain an unconditional basic sequence either. For if it did, then $X \oplus X$ would in particular contain a real unconditional sequence, spanning a real subspace $Y$. And since some subspace of $Y$ must either embed into the first or second summand of the decomposition $X \oplus X, X$ itself would contain a real unconditional basic sequence, contradicting our assumption.

Now, as mentioned in Section 2.2, if $T$ is an almost finite-dimensional isometry of $X, \hat{T}$ is an almost finite-dimensional isometry of $\hat{X}$, which then must be finite-dimensional. Since $F_{\hat{T}}=F_{T}+i F_{T}$, we finally conclude that also $F_{T}$ is finitedimensional.

Combining Theorem 5.10 and Theorem 6.4, we also obtain the following.

\section{COROLLARY 6.5}

Let $X$ be a Banach space with separable dual and not containing an unconditional basic sequence. Let $G \leq \mathrm{GL}_{a f}(X)$ be a bounded subgroup, and assume that no nonzero point of $X$ has a totally bounded $G$-orbit. Then $G$ is discrete and locally finite in the norm topology.

Proof

Since $\mathrm{GL}_{a f}(X)$ is norm closed in $\operatorname{GL}(X)$, by replacing $G$ by $\bar{G}^{\|\cdot\|}$ we may assume that $G$ is norm closed. Also, by renorming $X$, we can suppose that $G \leq \operatorname{Isom}_{a f}(X)$. Then, since by Theorem $6.4, G \leq \operatorname{Isom}_{f}(X)$, Theorem 5.10 applies.

\subsection{Groups of isometries in spaces with few operators}

We now combine the results of the previous sections in order to obtain a description of the group of isometries of a separable reflexive space $X$ with a small algebra of oper- 
ators. In this case, Proposition 3.5 will ensure that the norm and the strong operator topology coincide on $\operatorname{Isom}(X)$.

We recall that an infinite-dimensional Banach space is decomposable if it can be written as the direct sum of two infinite-dimensional subspaces and hereditarily indecomposable (HI) if it has no decomposable subspace. The first construction of an HI space was given by Gowers and Maurey in [35] to solve the unconditional basic sequence problem, since it is clear that an HI space cannot contain any subspace with an unconditional basis. Furthermore, Gowers and Maurey proved that any operator on a complex HI space is a strictly singular perturbation of a multiple of the identity. We will call this latter property of a (real or complex) Banach space the $(\lambda \mathrm{Id}+S)$-property. Note that the $(\lambda \mathrm{Id}+S)$-property easily implies that the space is indecomposable, since no projection with infinite-dimensional range and corange is of the form $\lambda \mathrm{Id}+S$, with $S$ strictly singular.

As an immediate consequence of Theorem 6.4, we have the following.

\section{THEOREM 6.6}

Let $X$ be a Banach space with the $(\lambda \operatorname{Id}+S)$-property and containing no unconditional basic sequence. Then each individual isometry acts nearly trivially on $X$.

COROLLARY 6.7

Let $X$ be a separable reflexive space with the $(\lambda \mathrm{Id}+S)$-property and containing no unconditional basic sequence. Assume that $X$ does not have a Schauder basis. Then Isom $(X)$ acts nearly trivially on $X$.

Proof

Note that, depending on whether $X$ is complex or real, we have by Theorem 6.6 that

$$
\operatorname{Isom}(X)=\mathbb{T} \times \operatorname{Isom}_{f}(X)
$$

or

$$
\operatorname{Isom}(X)=\{-1,1\} \times \operatorname{Isom}_{f}(X) .
$$

Thus, by Proposition 3.5, the norm and strong operator topologies coincide on $\operatorname{Isom}(X)$. So, as $\operatorname{Isom}_{f}(X)$ is norm closed in $\operatorname{Isom}(X)$, it is strongly closed in $\operatorname{Isom}(X)$ and hence also in GL(X). By Corollary 4.15, we see that $\operatorname{Isom}_{f}(X)$ and thus also $\operatorname{Isom}(X)$ acts nearly trivially on $X$.

COROLLARY 6.8

Let $X$ be a separable, reflexive, complex HI space without a Schauder basis. Then Isom $(X)$ acts nearly trivially on $X$. 
Of course, in the case when $X$ is permitted to have a Schauder basis, Theorem 6.6 does not itself describe the global action of the group Isom $(X)$, but with some extra hypotheses, we can get more information. Observe first that if $X$ is an infinitedimensional space with the $(\lambda \mathrm{Id}+S)$-property, then $\mathrm{GL}(X)=\mathbb{K}^{\star} \times \mathrm{GL}_{s}(X)$.

\section{THEOREM 6.9}

Let $X$ be a separable, infinite-dimensional, reflexive space with the $(\lambda \mathrm{Id}+$ $S$ )-property and containing no unconditional basic sequence. Assume that $G \leq$ $\mathrm{GL}_{S}(X)$ is a bounded subgroup. Then either

(i) $\quad G$ acts nearly trivially on $X$, or

(ii) $X$ admits a $G$-invariant decomposition $F \oplus H$, where $F$ is finite-dimensional and $\left.G\right|_{H}=\left\{\left.T\right|_{H} \mid T \in G\right\}$ is a countable, discrete, locally finite subgroup of $\mathrm{GL}_{f}(H)$, none of whose nonzero orbits are relatively compact; moreover, in this case, $X$ has a Schauder basis.

\section{Proof}

By Theorem 6.4, $G \leq \mathrm{GL}_{f}(X)$. Also, as in the proof of Corollary 6.7, we see that $\operatorname{Isom}_{f}(X)$ is strongly closed in $\operatorname{GL}(X)$. By renorming, we may assume that $G \leq$ $\operatorname{Isom}_{f}(X)$, whence also $M=\bar{G}^{\text {SOT }}$ is a subgroup of $\operatorname{Isom}_{f}(X)$. Applying Theorem 5.11 to $M$, we obtain a decomposition

$$
X=X_{1} \oplus X_{2} \oplus X_{3} \oplus X_{4}
$$

Here either $X_{1}=\{0\}$, in which case (i) holds, or $X_{1}$ has a complemented subspace with a Schauder basis.

By the indecomposability of $X$, in the latter case, $X$ itself has a Schauder basis, $H=X_{1}$ has finite codimension in $X$, and $F=X_{2} \oplus X_{3} \oplus X_{4}$ is finite-dimensional. Furthermore, $\left.M\right|_{X_{1}}=\left\{\left.T\right|_{X_{1}} \mid T \in M\right\}$ is a strongly closed subgroup of $\operatorname{GL}\left(X_{1}\right)$ contained in $\operatorname{Isom}_{f}\left(X_{1}\right)$ such that no nonzero $M$-orbit on $X_{1}$ is relatively compact. By Proposition 3.5, the strong operator and norm topologies coincide on $\left.M\right|_{X_{1}}$, while, by Theorem 5.10, $\left.M\right|_{X_{1}}$ is countable, discrete, and locally finite. It follows that $\left.G\right|_{X_{1}}=\left.M\right|_{X_{1}}$.

While the real HI space constructed by Gowers and Maurey has the $(\lambda \mathrm{Id}+$ $S$ )-property, this does not generalize to all real HI spaces (see [28]). Nor is it true that the complexification of a real HI space is complex HI (see [28] and [33, Proposition 3.16]). So it is not clear whether Corollary 6.8 extends to the real case.

Our methods may also be used in spaces with the $(\lambda \mathrm{Id}+S)$-property containing unconditional basic sequences. Such spaces do exist (see, e.g., [5]). 
THEOREM 6.10

Let $X$ be a separable reflexive space with the $(\lambda \mathrm{Id}+S)$-property. Then either Isom $(X)$ acts nearly trivially on $X$, or $X$ admits an isometry-invariant decomposition $X=F \oplus Y$, where $F$ is finite-dimensional and where no orbit of a nonzero point of $Y$ under $\operatorname{Isom}(X)$ is totally bounded.

Proof

By Theorem 3.4, depending on whether $X$ is complex or real, we have that

$$
\operatorname{Isom}(X)=\mathbb{T} \times \operatorname{Isom}_{a f}(X)
$$

or

$$
\operatorname{Isom}(X)=\{-1,1\} \times \operatorname{Isom}_{a f}(X),
$$

and so Proposition 3.5 applies to deduce that the strong operator and the norm topologies coincide on $\operatorname{Isom}(X)$, whence $G=\operatorname{Isom}_{a f}(X)$ is strongly closed in $\operatorname{GL}(X)$.

By Theorem 4.10, we have a $G$-invariant decomposition $X=K_{G} \oplus\left(K_{G}^{*}\right)^{\perp}$, where either $X=K_{G}$ or $K_{G}$ has infinite codimension. Furthermore, $X$ is indecomposable, so either $X=K_{G}$ or $K_{G}$ is finite-dimensional. In the first case, $G$ is almost periodic and thus by Theorem 3.11 acts nearly trivially on $X$. In the second case, we define $F=K_{G}$ and $Y=\left(K_{G}^{*}\right)^{\perp}$.

\section{Maximality and transitivity in spaces with few operators}

Let us begin by reviewing the various types of norms defined and studied by Pełczyński and Rolewicz in [52] and [58].

\section{Definition 7.1}

Let $(X,\|\cdot\|)$ be a Banach space, and, for any $x \in S_{X}$, let $\mathcal{O}(x)$ denote the orbit of $x$ under the action of $\operatorname{Isom}(X,\|\cdot\|)$. The norm $\|\cdot\|$ on $X$ is

(i) transitive if for any $x \in S_{X}, \mathcal{O}(x)=S_{X}$;

(ii) almost transitive if for any $x \in S_{X}, \mathcal{O}(x)$ is dense in $S_{X}$;

(iii) convex transitive if for any $x \in S_{X}, \operatorname{conv} \mathcal{O}(x)$ is dense in $B_{X}$;

(iv) uniquely maximal if whenever $\|\cdot\| \|$ is an equivalent norm on $X$ such that $\operatorname{Isom}(X,\|\cdot\|) \leq \operatorname{Isom}(X,\|\cdot\|)$, then $\|\cdot\| \|$ is a scalar multiple of $\|\cdot\|$;

(v) maximal if whenever $\|\cdot\| \|$ is an equivalent norm on $X$ such that $\operatorname{Isom}(X$, $\|\cdot\|) \leq \operatorname{Isom}(X,\|\cdot\|)$, then $\operatorname{Isom}(X,\|\cdot\|)=\operatorname{Isom}(X,\|\cdot\|)$.

Here, the implications (i) $\Rightarrow$ (ii) $\Rightarrow$ (iii) as well as (iv) $\Rightarrow$ (v) are obvious. Furthermore, Rolewicz [58] proved that any convex-transitive norm must be uniquely maximal, and E. R. Cowie [17] later reversed this implication by showing that a uniquely maximal norm is convex transitive. So (i) $\Rightarrow$ (ii) $\Rightarrow$ (iii) $\Leftrightarrow($ iv) $\Rightarrow(v)$. 
Almost transitive norms are not too difficult to obtain. For example, the classical norm on $L_{p}([0,1]), 1 \leq p<\infty$, is almost transitive (see [36], [58]). It is also known that the nontrivial ultrapower of a space with an almost transitive norm will have a transitive norm. There are therefore many examples of nonseparable, non-Hilbertian spaces with a transitive norm.

Rolewicz [58] also proved that if a space has a 1-symmetric basic sequence, then the norm is maximal. Therefore, the usual norms on the spaces $c_{0}$ and $\ell_{p}, 1 \leq p<\infty$, are maximal, though they are not convex transitive unless $p=2$.

More interesting than asking whether a specific norm on a Banach space $X$ has one of the above forms of transitivity or maximality is the question of whether $X$ admits an equivalent norm with these properties. In this direction, Becerra Guerrero and Rodríguez-Palacios [8, Theorem 6.8 and Corollary 6.9] showed the following interesting fact.

THEOREM 7.2 (Becerra Guerrero and Rodríguez-Palacios)

Suppose that $X$ is either Asplund or has the Radon-Nikodym property and that the norm $\|\cdot\|$ of $X$ is convex transitive. Then $\|\cdot\|$ is almost transitive, uniformly convex, and uniformly smooth. In particular, $X$ is super-reflexive.

This gives a list of spaces with no equivalent convex-transitive norm, $c_{0}, \ell_{1}$, Tsirelson's space $T$, Schlumprecht's space $S$, and Gowers-Maurey's space GM, for example.

Lusky [46] proved that every separable Banach space $Y$ is 1-complemented in some almost transitive separable space. So there are many different spaces with almost transitive norms, and, depending on the choice of $Y$, examples without a Schauder basis. Note, however, that Lusky's theorem cannot be improved to include reflexivity; that is, if $Y$ is reflexive but not super-reflexive, then, by Theorem 7.2, $Y$ does not even embed into a reflexive space with a convex-transitive norm.

However, the question of whether any super-reflexive space admits an equivalent almost transitive norm has remained open hitherto. This question is due to Deville, Godefroy, and Zizler [21, p. 176, Problem IV.2]. Based on results by Finet [30, p. 89], they observed that a positive answer would imply that if a Banach space $X$ has an equivalent norm with modulus of convexity of type $p \geq 2$ and another equivalent norm with modulus of smoothness of type $1 \leq q \leq 2$, then $X$ has an equivalent norm with both of these properties, which would be exceedingly useful in renorming theory. However, as we will see, this approach does not work.

We now answer the question of Deville, Godefroy, and Zizler along with Wood's problems of whether any Banach space has an equivalent maximal norm or even whether any bounded subgroup $G \leq \mathrm{GL}(X)$ is contained in a maximal bounded subgroup. 
LEMMA 7.3

There is a separable, infinite-dimensional, super-reflexive, complex HI space without a Schauder basis.

Proof

Recall that for a space $X$, if we define

$$
p(X)=\sup \{t \leq 2 \mid X \text { has type } t\}
$$

and

$$
q(X)=\inf \{c \geq 2 \mid X \text { has cotype } c\},
$$

then $X$ is said to be near-Hilbert if $p(X)=q(X)=2$. Now, by [27], there exists a uniformly convex and therefore super-reflexive complex HI space $X$ with a Schauder basis. Moreover, by [27], for any $1<p<2, X$ may be chosen so that for any finite sequence $x_{1}, \ldots, x_{n}$ of successive normalized vectors on the basis of $X$, we have

$$
\left\|x_{1}+\cdots+x_{n}\right\| \geq \frac{n^{1 / p}}{\log _{2}(n+1)},
$$

which implies that $X$ does not have type more than $p$. In particular, $X$ is not nearHilbert, and by classical results of Szankowski [64] (see also [45, Theorem 1.g.6]), this implies that some subspace $Y$ of $X$ does not have the approximation property and therefore fails to have a Schauder basis. On the other hand, $Y$ is still super-reflexive and $\mathrm{HI}$.

More precise estimates about type and cotype are given in [16] and imply that for any choice of parameters in the construction of [27], the space $X$ is not near-Hilbert and therefore has a subspace without the approximation property. On the other hand, it is also proved in [16] that for any $\epsilon>0$, the space $X$ (and therefore $Y$ as well) may be chosen to have type $2-\epsilon$ and cotype $2+\epsilon$.

PROPOSITION 7.4

Let $X$ be an infinite-dimensional Banach space with norm $\|\cdot\|$. Assume that $\operatorname{Isom}(X$, $\|\cdot\|)$ acts nearly trivially on $X$. Then $\|\cdot\|$ is not maximal.

\section{Proof}

Let $\|\cdot\|$ be such a norm on $X$, and let $X=F \oplus H$ be the associated decomposition for which $F$ is finite-dimensional and $\operatorname{Isom}(X)$ acts trivially on $H$. We fix a norm 1 vector $x_{0}$ in $H$, write $H$ as a direct sum $H=\left[x_{0}\right] \oplus M$, and define an equivalent 
norm $\|\cdot\| \mid$ on $X$ by

$$
\|\| f+\alpha x_{0}+m\||=\|f\|+| \alpha \mid+\| m \|,
$$

when $f \in F, \alpha$ is scalar, and $m \in M$.

If $T$ is an isometry for $\|\cdot\|$, then $\left.T\right|_{H}=\lambda \operatorname{Id}_{H}$ with $|\lambda|=1$, and so the equalities

$$
\left\|T\left(f+\alpha x_{0}+m\right)\right\|\left|=\left\|T f+\alpha \lambda x_{0}+\lambda m\right\|=\|T f\|+\right| \alpha \mid+\|m\|
$$

and

$$
\left\|f+\alpha x_{0}+m\right\|=\|f\|+|\alpha|+\|m\|
$$

show that $T$ is also a $\|\cdot \mid\|$-isometry. Therefore, any $\|\cdot\|$-isometry is a $\|\cdot \mid\|$-isometry.

Furthermore, the map $L$ on $X$ defined by

$$
L\left(f+\alpha x_{0}+m\right)=f-\alpha x_{0}+m
$$

is a $\|\cdot \mid\|$-isometry but not a $\|\cdot\|$-isometry, since there is no scalar $\lambda$ for which $\left.L\right|_{H}=$ $\lambda \operatorname{Id}_{H}$. This shows that $\|\cdot\|$ is not maximal.

\section{THEOREM 7.5}

There exists a separable, super-reflexive, complex Banach space $X$ that admits no equivalent maximal norm. In fact, if $\|\cdot\|$ is any equivalent norm on $X$, then $\operatorname{Isom}(X$, $\|\cdot\|)$ acts nearly trivially on $X$ and thus is a compact Lie group.

\section{Proof}

Let $X$ be the space given by Lemma 7.3, and notice that, by Corollary 6.8, the isometry group acts nearly trivially on $X$ for any equivalent norm. In particular, by Proposition 7.4, $X$ cannot have an equivalent maximal norm.

The above example is, of course, particularly strong since, for every equivalent norm, the isometry group is compact. So one may suspect there to be weaker counterexamples to the problem of Deville, Godefroy, and Zizler among more classical spaces.

In the real case, we obtain the following counterexample to Wood's questions, which, however, is not super-reflexive.

\section{THEOREM 7.6}

There exists a real, separable, reflexive Banach space with no equivalent maximal norm. 


\section{Proof}

The reflexive HI space GM of Gowers and Maurey [35] does not have type $p>1$. Therefore, the results of Szankowski [64] (see also [45, Theorem 1.g.6]) imply that some subspace $Y$ of GM fails to have a Schauder basis. Furthermore, $Y$ contains no unconditional basic sequence and, since by [35] every operator from a subspace of $X$ into $X$ is a strictly singular perturbation of a multiple of the inclusion map, $Y$ satisfies the $(\lambda \mathrm{Id}+S)$-property. The result then follows from Theorem 6.7 and Proposition 7.4.

In connection with this, we should mention the following conjecture due to Jarosz [39, p. 54].

\section{CONJECTURE 7.7 (K. Jarosz)}

Suppose that $G$ is a group and that $X$ is a real Banach space with $\operatorname{dim} X \geq|G|$. Then $X$ admits an equivalent renorming such that $\operatorname{Isom}(X) \cong\{-1,1\} \times G$.

In [29] this was verified for finite groups $G$ and separable spaces $X$, but, as we will see, the conjecture fails for infinite $G$.

\section{PROPOSITION 7.8}

If $X$ is the real HI space considered in Theorem 7.6, then for any norm on $X$, the group of isometries on $X$ is either finite or of cardinality $2^{\aleph_{0}}$. In particular, Jarosz's conjecture does not hold in general.

\section{Proof}

Assume that $X$ is the real HI space considered in Theorem 7.6. Then $\operatorname{Isom}(X)$ acts nearly trivially on $X$ and hence is a compact Lie group. It follows that $\operatorname{Isom}(X)$ is either finite or of size $2^{\boldsymbol{N}_{0}}$.

\section{Questions and further comments}

\subsection{Problems concerning spaces with few operators}

We suspect that the usage of reflexivity and the nonexistence of a Schauder basis in Theorem 7.5 are not necessary. That is, we conjecture the following.

\section{CONJECTURE 8.1}

Let $X$ be a complex HI space. Then the group of isometries acts nearly trivially on $X$.

A few comments on this conjecture are in order. First of all, the following result was essentially proved by Cabello-Sánchez [14, Theorem 2]. As we state a slightly 
more general result than in [14], we give the proof of the theorem for the sake of completeness.

\section{THEOREM 8.2 (Cabello-Sánchez)}

Let $G=\mathbb{T} \times \operatorname{Isom}_{f}(X)$ (resp., $G=\{-1,1\} \times \operatorname{Isom}_{f}(X)$ ) be the group of nearly trivial isometries of a space $X$. If the action of $G$ on $X$ is transitive, then the norm of $X$ is Euclidean, that is, $X$ is isometric to Hilbert space.

\section{Proof}

By transitivity, the only $\operatorname{Isom}_{f}(X)$-invariant subspaces of $X$ are the trivial ones. Therefore, by [14, Lemma 2], there exists an $\operatorname{Isom}_{f}(X)$-invariant inner product $\langle\cdot, \cdot\rangle$ on $X$ and some $x_{0} \in S_{X}$ such that $\left\langle x_{0}, x_{0}\right\rangle=1$. Now, if $T \in G$ and $\lambda \in \mathbb{T}$ is chosen such that $U=\lambda^{-1} T \in \operatorname{Isom}_{f}(X)$, then

$$
\left\langle T x_{0}, T x_{0}\right\rangle=\left\langle\lambda U x_{0}, \lambda U x_{0}\right\rangle=|\lambda|^{2}\left\langle U x_{0}, U x_{0}\right\rangle=1
$$

as well. By transitivity of $G$, this implies that $\langle x, x\rangle=\|x\|^{2}$ for all $x \in X$, which proves the theorem.

So by Theorem 6.6, we have the following.

\section{COROLLARY 8.3}

Let $X$ be a space with the $(\lambda \mathrm{Id}+S)$-property and without an unconditional basic sequence. Then no equivalent norm on $X$ is transitive.

As a tool toward proving Conjecture 8.1, it may be interesting to observe that the spectrum $\sigma(T)$ depends continuously on $T$ when $T$ belongs to $\operatorname{Isom}_{f}(X)$. For this, when $X$ a complex space and $T \in \operatorname{Isom}_{f}(X)$, we denote by $F_{\lambda}(T)$ the image of the spectral projection associated to the eigenvalue $\lambda$, and note that $F_{\lambda}(T)=$ $\operatorname{ker}(T-\lambda \mathrm{Id})$ by Gelfand's theorem. So, by Lemma 3.9, $F_{T}=\bigoplus_{\lambda \neq 1} F_{\lambda}(T)$ and $H_{T}=F_{1}(T)$.

\section{LEMMA 8.4}

Suppose that $X$ is a complex space and that $T \in \operatorname{Isom}_{f}(X)$. Then if $P_{i}$ denotes the canonical projection of $X$ onto $F_{\lambda_{i}}(T)$ corresponding to the decomposition

$$
X=F_{\lambda_{1}}(T) \oplus \cdots \oplus F_{\lambda_{m}}(T)
$$

we have $\left\|P_{i}\right\|=1$ for every $i$. Since $F_{T}$ is the complement of $H_{T}=F_{1}(T)$, it follows that the projection onto $F_{T}$ has norm at most 2 . 


\section{Proof}

Let $p_{n}(t)=n^{-1}\left(1+t+\cdots+t^{n-1}\right)$. Since the operator $p_{n}\left(\lambda_{i}^{-1} T\right)$ has norm at most 1 and acts as the identity on $F_{\lambda_{i}}(T)$, we have for any $x \in F_{\lambda_{i}}(T)$ and $y \in$ $\bigoplus_{j \neq i} F_{\lambda_{j}}(T)$,

$$
\left\|x+p_{n}\left(\lambda_{i}^{-1} T\right)(y)\right\|=\left\|p_{n}\left(\lambda_{i}^{-1} T\right)(x+y)\right\| \leq\|x+y\| .
$$

Note also that

$$
\left(\operatorname{Id}-\lambda_{i}^{-1} T\right) p_{n}\left(\lambda_{i}^{-1} T\right)=n^{-1}\left(\operatorname{Id}-\lambda_{i}^{-n} T^{n}\right) .
$$

Since $T-$ Id is injective on $\bigoplus_{j \neq i} F_{\lambda_{j}}(T)$ and therefore also invertible on $\bigoplus_{j \neq i} F_{\lambda_{j}}(T)$, we have for $y \in \bigoplus_{j \neq i} F_{\lambda_{j}}(T)$,

$$
p_{n}\left(\lambda_{i}^{-1} T\right)(y)=n^{-1}\left[\left.\left(\operatorname{Id}-\lambda_{i}^{-1} T\right)\right|_{j \neq i} F_{\lambda_{j}}(T)\right]^{-1}\left(\operatorname{Id}-\lambda_{i}^{-n} T^{n}\right)(y),
$$

whereby since $\left(\lambda_{i}^{-n} T^{n}\right)_{n}$ is bounded, $\lim _{n \rightarrow \infty} p_{n}\left(\lambda_{i}^{-1} T\right)(y)=0$. Applying this to the inequality $\left\|x+p_{n}\left(\lambda_{i}^{-1} T\right)(y)\right\| \leq\|x+y\|$, we get $\left\|P_{i}(x+y)\right\|=\|x\| \leq$ $\|x+y\|$.

\section{LEMMA 8.5}

Suppose that $X$ is a complex space. Then for any $S, T \in \operatorname{Isom}_{f}(X), \lambda \in \sigma(T)$, and $\mu_{1}, \ldots, \mu_{k} \in \sigma(S)$, if

$$
F_{\lambda}(T) \cap \bigoplus_{i=1}^{k} F_{\mu_{i}}(S) \neq\{0\}
$$

then

$$
\min _{i}\left|\mu_{i}-\lambda\right| \leq k\|S-T\| .
$$

It follows that for any $S, T \in \operatorname{Isom}_{f}(X)$ and $\lambda \in \sigma(T)$,

$$
\operatorname{dim} F_{\lambda}(T) \leq \operatorname{dim} \bigoplus_{|\mu-\lambda| \leq|\sigma(S)| \cdot\|S-T\|} F_{\mu}(S) .
$$

\section{Proof}

Suppose that $x_{i} \in F_{\mu_{i}}(S)$ and that $x_{1}+\cdots+x_{k} \in F_{\lambda}(T)$. Then, by Lemma 8.4, we have

$$
\begin{aligned}
\min _{i}\left|\mu_{i}-\lambda\right| \cdot\left\|x_{1}+\cdots+x_{k}\right\| & \leq \min _{i}\left|\mu_{i}-\lambda\right|\left(\left\|x_{1}\right\|+\cdots+\left\|x_{k}\right\|\right) \\
& \leq k \min _{i}\left|\mu_{i}-\lambda\right| \cdot \max _{j}\left\|x_{j}\right\|
\end{aligned}
$$




$$
\begin{aligned}
& \leq k \max _{j}\left\|\left(\mu_{j}-\lambda\right) x_{j}\right\| \\
& \leq k\left\|\left(\mu_{1}-\lambda\right) x_{1}+\cdots+\left(\mu_{k}-\lambda\right) x_{k}\right\| \\
& =k\left\|S\left(x_{1}+\cdots+x_{k}\right)-T\left(x_{1}+\cdots+x_{k}\right)\right\| \\
& \leq k\|S-T\| \cdot\left\|x_{1}+\cdots+x_{k}\right\| .
\end{aligned}
$$

Dividing by $\left\|x_{1}+\cdots+x_{k}\right\|$, we get $\min _{i}\left|\mu_{i}-\lambda\right| \leq k\|S-T\|$.

Now, suppose toward a contradiction that $A=\{\mu \in \sigma(S)|| \mu-\lambda|\leq| \sigma(S) \mid$. $\|S-T\|\}$ and that

$$
\operatorname{dim} F_{\lambda}(T)>\operatorname{dim} \bigoplus_{\mu \in A} F_{\mu}(S)
$$

Then, as $X=\bigoplus_{\mu \in \sigma(S)} F_{\mu}(S)$, we must have

$$
F_{\lambda}(T) \cap \bigoplus_{\mu \in \sigma(S) \backslash A} F_{\mu}(S) \neq\{0\}
$$

and so $|\mu-\lambda| \leq|\sigma(S)| \cdot\|S-T\|$ for some $\mu \in \sigma(S) \backslash A$, which is absurd.

LEMMA 8.6

Let $\mathcal{K}(\mathbb{T})$ denote the set of compact subsets of $\mathbb{T}$ equipped with the Vietoris topology, that is, the topology induced by the Hausdorff metric, and let $X$ be a complex space. Then $T \mapsto \sigma(T)$ is norm continuous as a map from $\operatorname{Isom}_{f}(X)$ to $\mathcal{K}(\mathbb{T})$.

\section{Proof}

Using that $\mathrm{GL}(X)$ is a norm-open subset of $\mathscr{L}(X)$, we see that if $S_{n} \underset{n \rightarrow \infty}{\longrightarrow} T$ and $\lambda_{n} \underset{n \rightarrow \infty}{\longrightarrow} \lambda$ for $\lambda_{n} \in \sigma\left(S_{n}\right)$, then also $\lambda \in \sigma(T)$. It follows that if $U \subseteq \mathbb{T}$ is open and $\sigma(T) \subseteq \mathcal{U}$, then $\sigma(S) \subseteq \mathcal{U}$ for all $S$ in a neighborhood of $T$. So $T \mapsto \sigma(T)$ is at least semicontinuous.

For the other half of continuity, note first that by Lemma 5.7 there is some $N$ such that $|\sigma(S)| \leq N$ for all $S$ in some neighborhood $\mathcal{N}$ of $T$. Therefore, by Lemma 8.5, for all $S \in \mathcal{N}$ and $\lambda \in \sigma(T)$,

$$
\min _{\mu \in \sigma(S)}|\mu-\lambda| \leq N\|S-T\| .
$$

It follows that if $U \subseteq \mathbb{T}$ is open and $\sigma(T) \cap \mathcal{U} \neq \emptyset$, then also $\sigma(S) \cap \mathcal{U} \neq \emptyset$ for all $S$ in a neighborhood of $T$, finishing the proof of continuity.

Finally, several examples of nonreflexive HI spaces have been considered in the literature. Gowers [34] constructed a separable HI space such that every subspace has 
nonseparable dual, and Argyros, Arvanitakis, and Tolias [3] constructed a nonseparable, (necessarily nonreflexive) HI space. Also, the Argyros-Haydon space AH (see [4]) is $\mathrm{HI}$ and has dual isomorphic to $\ell_{1}$. As noted, any single isometry of a complex HI space $X$ must act nearly trivially on $X$. But nothing is known about the global behavior of $\operatorname{Isom}(X)$ even in the above-mentioned examples.

\subsection{Problems concerning Hilbert space}

As mentioned in the introduction, the following strong versions of the second part of Mazur's rotation problem remain open.

\section{Problem 8.7}

Suppose that $\|\cdot\|$ is an equivalent maximal or almost transitive norm on $\mathscr{H}$. Must $\|\cdot\|$ be Euclidean?

Also, though not every bounded subgroup $G \leq \mathrm{GL}(\mathscr{H})$ permits an equivalent $G$-invariant Euclidean norm, the following is open.

\section{Problem 8.8}

Suppose that $G \leq \operatorname{GL}(\mathscr{H})$ is a bounded subgroup. Must there be an equivalent $G$-invariant maximal, almost transitive, or even transitive norm on $\mathscr{H}$ ?

Note that by Theorem 7.2, convex transitivity coincides with almost transitivity on $\mathscr{H}$.

In [13] it is mentioned that if $X$ is a space with an almost transitive norm and there exists an isometry $\mathrm{Id}+F$ for which $F$ has rank 1 , then $X$ is isometric to a Hilbert space. However, the following question is still open.

\section{Problem 8.9 (Cabello-Sánchez)}

Let $X$ be a space with an (almost) transitive norm and which admits a nontrivial finite-dimensional isometry. Must $X$ be Hilbertian?

Note that, by Corollary 4.14, if $X$ is separable reflexive and satisfies the hypothesis, then $X$ must have a Schauder basis.

\subsection{General questions}

Of course, to prove Conjecture 8.1, one would like to improve on Theorem 5.11. For this, the following would be a intermediate step. 
Problem 8.10

Let $X$ be a separable Banach space, let $G \leq \operatorname{Isom}_{f}(X)$, and assume that

$$
\sup \left\{\operatorname{dim} F_{T} \mid T \in G\right\}<\infty .
$$

Must $G$ act nearly trivially on $X$ ?

By Proposition 3.10, this is equivalent to asking whether $G$ is relatively norm compact. On the other hand, for a potential counterexample, one might begin with the following.

\section{Problem 8.11}

Find a separable space $X$ and a bounded subgroup of $\mathrm{GL}_{f}(X)$ which is infinite and discrete for SOT.

We know of no real uniformly convex space for which no renorming is maximal. Considered as a real space, the example of Theorem 7.5 is also HI and uniformly convex, but does not satisfy the $(\lambda \mathrm{Id}+S)$-property.

\section{Problem 8.12}

Does there exist a real super-reflexive Banach space without a maximal norm? Without an almost transitive norm?

As mentioned, the fact that there exist complex super-reflexive spaces with no equivalent almost transitive norms shows that a certain approach to smooth renormings does not work. Our counterexamples are therefore candidates for a negative answer to the following question.

\section{Problem 8.13}

Does there exist a Banach space $X$ and constants $1 \leq q \leq 2 \leq p$ such that the set of equivalent norms on $X$ with modulus of convexity of type $p$ and the set of equivalent norms on $X$ with modulus of smoothness of type $q$ are disjoint and both nonempty? Is the space defined in [27] or one of its subspaces an example of this?

Note that if the norm on a space $X$ is convex transitive, then $X$ has no nontrivial isometry-invariant subspaces. Thus, if $X$ is also separable reflexive and admits a nontrivial finite-dimensional isometry, Corollary 4.14 implies that $X$ has a Schauder basis. It would be interesting to know if the existence of a finite-dimensional isometry can be removed; that is, we would be interested in the answer to the following problem. 


\section{Problem 8.14}

Let $X$ be a separable, reflexive, Banach space with a convex-transitive norm. Does it follow that $X$ has a Schauder basis?

Acknowledgments. We are grateful to J. Melleray, V. Pestov, and the anonymous referees for many insightful remarks.

\section{References}

[1] L. ALAOGLU and G. BIRKHOFF, General ergodic theorems, Ann. of Math. (2) 41 (1940), 293-309. MR 0002026. (1798)

[2] G. ALLEXANDROV, D. KUTZAROVA, and A. PLICHKO, A separable space with no Schauder decomposition, Proc. Amer. Math. Soc. 127 (1999), 2805-2806. MR 1670410. DOI 10.1090/S0002-9939-99-05370-8. (1802)

[3] S. A. ARGYROS, A. D. ARVANITAKIS, and A. G. TOLIAS, "Saturated extensions, the attractors method, and hereditarily James tree spaces" in Methods in Banach Space Theory, London Math. Soc. Lecture Note Ser. 337, Cambridge Univ. Press, Cambridge, 2006, 1-90. MR 2326379. DOI 10.1017/CBO9780511721366.003. (1825)

[4] S. A. ARGYROS and R. G. HAYDON, A hereditarily indecomposable $\mathscr{L}_{\infty}$-space that solves the scalar-plus-compact problem, Acta Math. 206 (2011), 1-54. MR 2784662. DOI 10.1007/s11511-011-0058-y. (1774, 1825)

[5] S. A. ARGYROS and A. MANOUSSAKIS, An indecomposable and unconditionally saturated Banach space, Studia Math. 159 (2003), 1-32. MR 2030739. DOI 10.4064/sm159-1-1. (1816)

[6] U. BADER, A. FURMAN, T. GELANDER, and N. MONOD, Property $(T)$ and rigidity for actions on Banach spaces, Acta Math. 198 (2007), 57-105. MR 2316269. DOI 10.1007/s11511-007-0013-0. (1773, 1796)

[7] S. BANACH, Théorie des opérations linéaires, reprint of the 1932 original, Éditions Jacques Gabay, Sceaux, 1993. MR 1357166. (1772)

[8] J. BECERRA GUERRERO and A. RODRÍGUEZ-PALACIOS, Transitivity of the norm on Banach spaces, Extracta Math. 17 (2002), 1-58. MR 1914238. (1773, 1818)

[9] S. F. BELLENOT, Banach spaces with trivial isometries, Israel J. Math. 56 (1986), 89-96. MR 0879916. DOI 10.1007/BF02776242. (1774)

[10] Y. BENYAMINI and J. LINDENSTRAUSS, Geometric Nonlinear Functional Analysis, Vol. 1, Amer. Math. Soc. Colloq. Publ. 48, Amer. Math. Soc., Providence, 2000. MR 1727673. (1777, 1778, 1796)

[11] J. F. BERGLUND, H. D. JUNGHENN, and P. MILNES, Analysis on Semigroups: Function Spaces, Compactifications, Representations, Canadian Mathematical Society Series of Monographs and Advanced Texts, John Wiley, New York, 1989. MR 0999922. (1797, 1799)

[12] P. L. BOWERS, Maximal convex metrics on some classical metric spaces, Geom. Dedicata 29 (1989), 125-132. MR 0988262. DOI 10.1007/BF00182113. (1773) 
[13] F. CABELLO-SÁNCHEZ, "Regards sur le problème des rotations de Mazur" in $I I$ Congress on Examples and Counterexamples in Banach Spaces (Badajoz, 1996), Extracta Math. 12 (1997), 97-116. MR 1607201. (1773, 1825)

[14] - A theorem on isotropic spaces, Studia Math. 133 (1999), 257-260. MR 1687219. (1821, 1822)

[15] J. W. CALKIN, Two-sided ideals and congruences in the ring of bounded operators in Hilbert space, Ann. of Math. (2) 42 (1941), 839-873. MR 0005790. (1784)

[16] J. M. F. CASTILLO, V. FERENCZI, and Y. MORENO, On uniformly finitely extensible Banach spaces, preprint, 2012. (1819)

[17] E. R. COWIE, A note on uniquely maximal Banach spaces, Proc. Edinb. Math. Soc. (2) 26 (1983), 85-87. MR 0695645. DOI 10.1017/S0013091500028091. (1817)

[18] M. M. DAY, Means for the bounded functions and ergodicity of the bounded representations of semi-groups, Trans. Amer. Math. Soc. 69 (1950), 276-291. MR 0044031. (1773)

[19] K. DE LEEUW and I. GLICKSBERG, Applications of almost periodic compactifications, Acta Math. 105 (1961), 63-97. MR 0131784. (1797)

[20] G. DEBS, G. GODEFROY, and J. SAINT-RAYMOND, Topological properties of the set of norm-attaining linear functionals, Canad. J. Math. 47 (1995), 318-329. MR 1335081. DOI 10.4153/CJM-1995-017-3. (1794)

[21] R. DEVILLE, G. GODEFROY, and V. ZIZLER, Smoothness and Renormings in Banach Spaces, Pitman Monogr. Surv. Pure Appl. Math. 64, John Wiley, New York, 1993. MR 1211634. (1774, 1777, 1779, 1791, 1792, 1793, 1794, 1795, 1818)

[22] J. DIXMIER, Les moyennes invariantes dans les semi-groupes et leurs applications, Acta Sci. Math. (Szeged) 12 (1950), 213-227. MR 0037470. (1773)

[23] N. DUNFORD and J. T. SCHWARTZ, Linear Operators, I: General Theory, Pure Appl. Math. 7, Interscience, New York, 1958. MR 0117523. (1777)

[24] L. EHRENPREIS and F. I. MAUTNER, Uniformly bounded representations of groups, Proc. Natl. Acad. Sci. USA 41 (1955), 231-233. MR 0071434. (1773)

[25] I. EPSTEIN and N. MONOD, Nonunitarizable representations and random forests, Int. Math. Res. Not. IMRN 2009, no. 22, 4336-4353. MR 2552305.

DOI 10.1093/imrn/rnp090. (1774)

[26] M. FABIAN, P. HABALA, P. HÁJEK, V. MONTESINOS, and V. ZIZLER, Banach Space Theory: The Basis for Linear and Nonlinear Analysis, CMS Books Math./Ouvrages Math. SMC, Springer, New York, 2011. MR 2766381. (1796)

[27] V. FERENCZI, A uniformly convex hereditarily indecomposable Banach space, Israel J. Math. 102 (1997), 199-225. MR 1489106. DOI 10.1007/BF02773800. (1776, 1819, 1826)

[28] - Uniqueness of complex structure and real hereditarily indecomposable Banach spaces, Adv. Math. 213 (2007), 462-488. MR 2331251. DOI 10.1016/j.aim.2007.01.003. (1816)

[29] V. FERENCZI and E. M. GALEGO, Countable groups of isometries on Banach spaces, Trans. Amer. Math. Soc. 362, no. 8 (2010), 4385-4431. MR 2608411. DOI 10.1090/S0002-9947-10-05034-8. (1775, 1784, 1821) 
[30] C. FINET, Uniform convexity properties of norms on a super-reflexive Banach space, Israel J. Math. 53 (1986), 81-92. MR 0861899. DOI 10.1007/BF02772671. (1818)

[31] R. J. FLEMING and J. E. JAMISON, Isometries on Banach Spaces, Vol. 2: Vector-Valued Function Spaces, Chapman \& Hall/CRC Monogr. Surv. Pure Appl. Math. 138, Chapman \& Hall/CRC, Boca Raton, Fla., 2008. MR 2361284. (1777)

[32] M. GONZÁLEZ, On essentially incomparable Banach spaces, Math. Z. 215 (1994), 621-629. MR 1269493. DOI 10.1007/BF02571733. (1784)

[33] M. GONZÁLEZ and J. M. HERRERA, Decompositions for real Banach spaces with small spaces of operators, Studia Math. 183 (2007), 1-14. MR 2360254.

DOI 10.4064/sm183-1-1. (1816)

[34] W. T. GOWERS, A Banach space not containing $c_{0}, \ell_{1}$ or a reflexive subspace, Trans. Amer. Math. Soc. 344, no. 1 (1994), 407-420. MR 1250820. DOI 10.2307/2154723. (1824)

[35] W. T. GOWERS and B. MAUREY, The unconditional basic sequence problem, J. Amer. Math. Soc. 6 (1993), 851-874. MR 1201238. DOI 10.2307/2152743. (1774, $1802,1814,1815,1821)$

[36] P. GREIM, J. E. JAMISON, and A. KAMINSKA, Almost transitivity of some function spaces, Math. Proc. Cambridge Philos. Soc. 116 (1994), 475-488. MR 1291754. DOI 10.1017/S0305004100072753. (1772, 1818)

[37] E. HILLE and R. S. PHILLIPS, Functional Analysis and Semi-Groups, Amer. Math. Soc. Colloq. Publ. 31, Amer. Math. Soc., Providence, 1957. MR 0089373. (1782)

[38] K. JACOBS, Fastperiodizitätseigenschaften allgemeiner Halbgruppen in Banach-Räumen, Math. Z. 67 (1957), 83-92. MR 0087057. (1797)

[39] K. JAROSZ, Any Banach space has an equivalent norm with trivial isometries, Israel J. Math. 64 (1988), 49-56. MR 0981748. DOI 10.1007/BF02767369. (1775, 1821)

[40] I. KAPLANSKY, Lie Algebras and Locally Compact Groups, reprint of the 1974 edition, Chicago Lectures in Math., Univ. Chicago Press, Chicago, 1995. MR 1324106. (1783)

[41] A. S. KECHRIS, Classical Descriptive Set Theory, Grad. Texts in Math. 156, Springer, New York, 1995. MR 1321597. DOI 10.1007/978-1-4612-4190-4. (1780, 1787)

[42] G. LANCIEN, Dentability indices and locally uniformly convex renormings, Rocky Mountain J. Math. 23 (1993), 635-647. MR 1226193. DOI 10.1216/rmjm/1181072581. (1795)

[43] J. LINDENSTRAUSS, On nonseparable reflexive Banach spaces, Bull. Amer. Math. Soc. (N.S.) 72 (1966), 967-970. MR 0205040. (1791)

[44] J. LINDENSTRAUSS and L. TZAFRIRI, Classical Banach Spaces, I, Ergeb. Math. Grenzgeb. 92, Springer, Berlin, 1977. MR 0500056. (1777, 1801)

[45] - Classical Banach Spaces, II, Ergeb. Math. Grenzgeb. 97, Springer, Berlin, 1979. MR 0540367. (1777, 1819, 1821)

[46] W. LUSKY, A note on rotations in separable Banach spaces, Studia Math. 65 (1979), 239-242. MR 0567078. (1818)

[47] S. MAZUR, Quelques propriétés caractéristiques des espaces euclidiens, C. R. Math. Acad. Sci. Paris 207 (1938), 761-764. (1773) 
[48] M. G. MEGRELISHVILI, "Operator topologies and reflexive representability" in Nuclear Groups and Lie Groups (Madrid, 1999), Res. Exp. Math. 24, Heldermann, Lemgo, 2001, 197-208. MR 1858149. (1780)

[49] N. MONOD and N. OZAWA, The Dixmier problem, lamplighters and Burnside groups, J. Funct. Anal. 258 (2010), 255-259. MR 2557962. DOI 10.1016/j.jfa.2009.06.029. (1774)

[50] R. NAGEL and F. RÄBIGER, Superstable operators on Banach spaces, Israel J. Math. 81 (1993), 213-226. MR 1231188. DOI 10.1007/BF02761307. (1813)

[51] A. J. OL'ŠHANSKIĬ, On the question of the existence of an invariant mean on a group (in Russian), Uspekhi Mat. Nauk 35 (1980), 199-200. MR 0586204. (1773)

[52] A. PEŁCZYŃSKI and S. ROLEWICZ, Best norms with respect to isometry groups in normed linear spaces, short communication on International Mathematical Congress in Stockholm (1964), 104. (1772, 1817)

[53] G. PISIER, Counterexamples to a conjecture of Grothendieck, Acta Math. 151 (1983), 181-208. MR 0723009. DOI 10.1007/BF02393206. (1802)

[54] - "Are unitarizable groups amenable?" in Infinite Groups: Geometric, Combinatorial and Dynamical Aspects, Progr. Math. 248, Birkhäuser, Basel, 2005, 323-362. MR 2195457. DOI 10.1007/3-7643-7447-0_8. (1774)

[55] F. RÄBIGER and W. J. RICKER, $C_{0}$-groups and $C_{0}$-semigroups of linear operators on hereditarily indecomposable Banach spaces, Arch. Math. (Basel) 66 (1996), 60-70. MR 1363778. DOI 10.1007/BF01323983. (1775, 1784)

[56] $-C_{0}$-semigroups and cosine families of linear operators in hereditarily indecomposable Banach spaces, Acta Sci. Math. (Szeged) 64 (1998), 697-706. MR 1666079. (1775, 1784)

[57] F. P. RAMSEY, On a problem of formal logic, Proc. Lond. Math. Soc. (2) 30 (1930), 264-286. MR 1576401. DOI 10.1112/plms/s2-30.1.264. (1782)

[58] S. ROLEWICZ, Metric Linear Spaces, 2nd ed., Polish Scientific, Warsaw, 1984. MR 0802450. (1772, 1817, 1818)

[59] C. RYLL-NARDZEWSKI, Generalized random ergodic theorems and weakly almost periodic functions, Bull. Acad. Polon. Sci. Sér. Math. Astronom. Phys. 10 (1962), 271-275. MR 0169984. (1796)

[60] P. V. SEMËNOV and A. I. SKORIK, Isometries of James spaces (in Russian), Mat. Zemetki 38 (1985), no. 4, 537-544, 635; English translation in Math. Notes 38 (1986), 804-808. MR 0819419. (1774)

[61] K. SHIGA, Representations of a compact group on a Banach space, J. Math. Soc. Japan 7 (1955), 224-248. MR 0082624. (1782)

[62] A. I. SHTERN, Norm continuous representations of locally compact groups, Russ. J. Math. Phys. 15 (2008), 552-553. MR 2470858.

DOI 10.1134/S1061920808040134. (1790)

[63] J. STERN, Le groupe des isométries d'un espace de Banach, Studia Math. 64 (1979), 139-149. MR 0537117. (1775)

[64] A. SZANKOWSKI, Subspaces without the approximation property, Israel J. Math. 30 (1978), 123-129. MR 0508257. DOI 10.1007/BF02760833. (1819, 1821) 
[65] B. SZŐKEFALVI-NAGY [DE SZ. NAGY], On uniformly bounded linear transformations in Hilbert space, Acta Univ. Szeged. Sect. Sci. Math. 11 (1947), 152-157. MR 0022309. (1773)

[66] J.-P. TROALLIC, Une approche nouvelle de la presque-périodicité faible, Semigroup Forum 22 (1981), 247-255. MR 0613094. DOI 10.1007/BF02572804. (1797)

[67] J. VON NEUMANN and E. P. WIGNER, Minimally almost periodic groups, Ann. of Math. (2) 41 (1940), 746-750. MR 0002891. (1777)

[68] G. V. WOOD, Maximal symmetry in Banach spaces, Proc. Roy. Irish Acad. Sect. A 82 (1982), 177-186. MR 0701377. (1774)

\section{Ferenczi}

Instituto de Matemática e Estatística, Universidade de São Paulo, Cidade Universitária, 05508-90 São Paulo, SP, Brazil; ferenczi@ime.usp.br

Institut de Mathématiques de Jussieu, Université Pierre et Marie Curie - Paris 6, 75252 Paris

Cedex 05, France; valentin.ferenczi@upmc.fr

\section{Rosendal}

Department of Mathematics, Statistics, and Computer Science (M/C 249), University of Illinois at Chicago, Chicago, Illinois 60607-7045, USA; rosendal.math@gmail.com 